\title{
Saturn's tropospheric particles phase function and spatial distribution from Cassini ISS 2010-11 observations
}

\author{
Santiago Pérez-Hoyos ${ }^{\mathrm{a}, \mathrm{b}}$, José Francisco Sanz-Requena ${ }^{\mathrm{c}}$, Agustín \\ Sánchez-Lavega ${ }^{\mathrm{a}, \mathrm{b}}$, Patrick G.J. Irwin ${ }^{\mathrm{d}}$, Andrew Smith ${ }^{\mathrm{d}}$ \\ ${ }^{a}$ Departamento de Física Aplicada I, ETS Ingeniería UPV/EHU, Alameda de Urquijo \\ s/n, 48013 Bilbao, Spain \\ ${ }^{b}$ Unidad Asociada Grupo Ciencias Planetarias UPV/EHU-IAA (CSIC), Bilbao, Spain \\ ${ }^{c}$ Departemento de Ciencias Experimentales, Universidad Europea Miguel de Cervantes, \\ C/Padre Julio Chevalier, 47012 Valladolid, Spain. \\ ${ }^{d}$ Atmospheric, Oceanic and Planetary Physics, University of Oxford, Clarendon \\ Laboratory, Parks Road, Oxford OX1 3PU, UK
}

\section{Abstract}

1 The phase function describes the way particles scatter the incoming radiation.

2 This is a fundamental piece of knowledge in order to understand how a plan-

3 etary atmosphere scatters sunlight and so it has a profound influence in the

4 retrieved atmospheric properties such as cloud height, particle density distri-

5 bution and radiative forcing by aerosols. In this work we analyze data from

6 the Imaging Science Subsystem (ISS) instrument onboard Cassini spacecraft

7 to determine the particle phase function at blue (451 nm) and near infrared

s wavelengths $(727-890 \mathrm{~nm}$ ) of particles in the upper troposphere, where most

9 of the incoming visible sunlight is scattered. In order to do so, we use obser-

10 vations taken in later 2010 and 2011 covering a broad range of phase angles

11 from $\sim 10^{\circ}$ to $\sim 160^{\circ}$ in the blue (BL1) and near infrared filters associated

12 with intermediate and deep methane absorption bands (MT2, CB2, MT3).

13 Particles at all latitudes are found to be strongly forward scattering. The

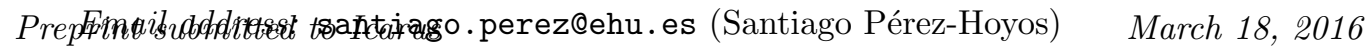


equatorial particles are in good agreement with laboratory measurements of $10 \mu \mathrm{m}$ ammonia ice crystals, while mid- and sub-polar latitude particles may be similar to the equatorial particles, but they may also be consistent with 1 $\mu \mathrm{m}$ ellipsoids with moderate aspect ratios. Uncertainties due to limited phase coverage and parameter degeneracy prevent strong constraints of the particle shapes and sizes at these locations.Results for the particle phase function are also used to describe the spatial distribution of tropospheric particles both vertically and latitudinally in the Northern hemisphere.

Keywords: Saturn, atmosphere; Radiative transfer; Atmospheres, structure

\section{Introduction}

The distribution of aerosols and particles in the upper troposphere and lower stratosphere of the giant planets is a fundamental piece of information in order to understand the way the atmospheres of these bodies behave. Aerosols act both as scatterers and as absorbers and both roles are essential. By reflecting the incoming sunlight, they serve as tracers for the atmospheric dynamics. These tracers allow the determination of the zonal wind profile (Vasavada et al., 2006; García-Melendo et al., 2011) and its local features (García-Melendo et al., 2010). They also reveal the presence of particular atmospheric structures (del Río-Gaztelurrutia et al., 2010), the most obvious ones being the periodic giant storms (often nicknamed Great White Spots, GWS hereafter) arising roughly every seasonal cycle, as seen in 1990 (Sánchez-Lavega et al., 1991, 1993) and 2010 (Fischer et al., 2011; SánchezLavega et al., 2011, 2012; Sayanagi et al., 2013). But aerosols forming cloud features do not just serve as passive tracers for the atmospheric dynamics, the 
combination of their absorption and scattering also has an influence in the energy budget of the planet as reviewed in Pérez-Hoyos and Sánchez-Lavega (2006b). Thus, the goal of this paper is to provide a better understanding of how the Saturnian tropospheric aerosols scatter and absorb solar radiation at visual wavelengths.

Briefly speaking, the evolution of our understanding of this topic (i.e. retrieval of vertical distribution of Saturn's upper tropospheric/lower stratospheric particles by means of reflected sunlight at visible wavelengths) is profoundly influenced by the advances in the spacecraft exploring Saturn and their particular orbits. The state of the art by the early 1980s is summarized by the work of Tomasko et al. (1984), in particular the use of methane bands in the near infrared for sounding the planet's atmosphere (West, 1983). Data from Pioneer 11's 1979 flyby of Saturn had provided our first opportunity for measuring the scattering phase function of aerosols in Saturn's atmosphere (Tomasko and Doose, 1984,1985$)$ at blue $(\sim 440 \mathrm{~nm})$ and red wavelengths $(\sim$ $640 \mathrm{~nm})$. The years after the launch of the Hubble Space Telescope provided additional information on general atmospheric properties (Pérez-Hoyos et al., 2005; Karkoschka and Tomasko, 2005) while other ground-based observatories (Temma et al., 2005) contributed to the topic. This, together with early work by the Cassini mission, was compiled by West et al. (2009). During the Cassini era, the most notable work on this topic is the study of Roman et al. (2013), which is based in a set of observations very similar to the ones used in this research, although taken some seven years earlier and restricted in phase angle coverage. Obviously, particle properties and related phenomena can also be studied at other wavelengths, e.g. the emitted thermal radiation 
as in West et al. (2009) or Fletcher et al. (2011a,b), but, all in all, if we want to understand the atmospheric regime as a whole we need to have a good information on where in the atmosphere the particules acting as tracers are located.

In spite of the advancement during the last 30 years, particularly due to the use of longer wavelengths to sound levels deeper and higher in the atmosphere than those sounded in the visible, the phase function used to describe the way the particles in the upper troposphere scatter incoming radiation is still the one determined by Tomasko and Doose (1984). The prevalence of the Pioneer's phase function is mainly due to the impossibility of observing Saturn at phase angles greater than $6^{\circ}$ from Earth. The phase angle is defined as the observer-Saturn-Sun angle and supplements the scattering angle. Thus, ground-based observations limited to low phase angles provide information mostly on the backscattering, while access to the forward scattering requires observations at high phase angles only attainable from spacecraft in orbit around the planet. Since the flybys in the early 1980s, no spacecraft has provided access to this information. During Saturn's seasonal cycle, the characteristic size or shape of the tropospheric particles could have been substantially changed thus modifying the particle phase function. This could in turn result in less accurate vertical cloud structure models and hence, the main goal of this paper is to constrain the particle phase function at least for a snapshot covering Saturn's Northern hemisphere during late 2010 and during 2011, comparing it with the early work based on Pioneer 11 photometry (Tomasko and Doose, 1984) in 1979. It must be noted that the Pioneer data were restricted to two broad bands, while Cassini ISS allows the use of narrow 
filters around methane bands which provide a better vertical resolution.

The paper is organized as follows: section 2 is devoted to a short description of the observations used in this work, as well as to the arguments used for latitude selection. Section 3 covers the radiative transfer model, including the description for the model atmosphere and its main parameters. The main results are shown in section 4 , first presenting the retrieved particle phase function for each latitude, then describing the vertical cloud structure as a function of latitude. A discussion on the meaning of the particle phase function can be found in section 5, as well as the dynamical implications of the retrieved distribution of particles. A summary of the main conclusions of this work is presented in section 6 .

\section{Observations}

\subsection{Overview of the observations}

The observations used in this work are summarized in table 1 . In this table, we show the date of each observing run together with the sub-spacecraft planetocentric latitude $B$ (close to the Equator for all cases), the sub-solar planetocentric latitude $B^{\prime}$ (ranging from $7^{\circ} \mathrm{N}$ to $12^{\circ} \mathrm{N}$ ) and phase angle $\alpha$, defined as explained above as the observer-planet-Sun angle (Sánchez-Lavega, 2011). There are 72 images taken in later 2010 and 2011 in a blue wide filter (BL1, 451nm) and three near infrared filters (Porco et al., 2004) which cover an intermediate methane band (MT2, 727nm), its adjacent continuum (CB2, $752 \mathrm{~nm}$ ) and a deep methane band (MT3, $890 \mathrm{~nm}$ ). The prime criterion for the image selection was to cover a wide range of phase angles for a time short enough not to display evident changes in the apparent reflectivity of 
the planet, more than those derived from the changing geometry, as it will be discussed in section 2.2. This proved to be a challenge, particularly in terms of latitude coverage, but 2011 offered a good sampling for the above mentioned filters for the Northern hemisphere. In the Equatorial Zone (latitudes below $20^{\circ} \mathrm{N}$ ) the phase angle covers from $\sim 10^{\circ}$ in August 2011 to $\sim 160^{\circ}$ in March 2011, which gives access to scattering angles from $\sim 20^{\circ}$ to $\sim 170^{\circ}$. Mid- and sub-polar latitudes, however, were not observed at this extreme phase angles and only reach $\sim 110^{\circ}$, therefore $\sim 70^{\circ}$ scattering angles. The same is true for BL1 observations, not including all phase angles. Figures 1 and 2 show cylindrically projected observations for all 18 dates in the three filters used here.

It must be noted that, in December 2010, a GWS erupted in Saturn's mid-latitudes (Fischer et al., 2011; Sánchez-Lavega et al., 2011), producing an intense disturbance of the atmosphere in a band roughly covering from $15^{\circ} \mathrm{N}$ to $40^{\circ} \mathrm{N}$ (Sánchez-Lavega et al., 2012; Sayanagi et al., 2013). For an analysis of the cloud structure of this phenomenon see Sanz-Requena et al. (2012) and Sromovsky et al. (2013). Obviously, those latitudes were not selectable for this research and there was a need for checking that the disturbance had not affected other latitudes.

One interesting point for the selected near infrared wavelengths is that they are close enough not to expect significant variations in the optical properties (e.g. single scattering albedos, real and imaginary refractive indices and phase functions), assuming that the spectral behaviour is smooth. So our first assumption is that particle properties must be constant over the 727-890 $\mathrm{nm}$ wavelength range. However, methane absorption varies strongly 
from one wavelength to another, thus probing a good range of altitudes in the upper troposphere. The exact sounding levels depend strongly on the vertical distribution of particles, but for a clear atmosphere they can be calculated to be between 60 mbar (MT3) down to almost 6 bar (CB2), as shown by Roman et al. (2013). Since the role of particles is about the same for all the infrared wavelengths, in a cloudy atmosphere the sounding levels will move upwards and will tend to squeeze the weighting functions making the average sounding levels closer in altitude (Sánchez-Lavega et al., 2007). Thus, the scattering properties are also assumed to be constant over the range of altitudes sensed by the near infrared filters. Note that this is not necessarily the case for the BL1 filter observations.

All the images were navigated using the PLIA software (Hueso et al., 2010) and photometrically calibrated to absolute reflectivity using Cassini Imaging Science Subsystem CALibration software (CISSCAL v3.6) described by Porco et al. (2004) and West et al. (2010). A full description of the ISS instrument can be found in fact in Porco et al. (2004). Here we will assume a constant relative error of $10 \%$ in absolute reflectivity for all data. This number is an intermediate estimate for the dispersion of results retrieved from observations of standard sources, as discussed in West et al. (2010), where a more thorough discussion on the sources of uncertainty can be found. In this work, all observations were transformed into 18 co-registered data cubes (with two spatial and one spectral directions) oversampled to a resolution of $0.1^{\circ}$ per pixel and including scattering angles for each spatial coordinate, as well as other useful meta data such as date and time of the observations or sub-solar and sub-spacecraft latitude and longitude. 


\subsection{Reflectivity ratios}

We show in figures 1 and 2 the reflectivity maps for all dates used in the following sections. All these maps are Lambert-corrected for limb-darkening, purely for representation purposes. This correction does not work equally well for all filters and latitudes, particularly for BL1 and CB2 observations which are not completely corrected with this approximation. The reflectivity as a function of wavelength and scattering angles is the observable that must be reproduced by the numerical modeling used in this paper; however, the changing geometry makes it difficult to assess the stability of the atmosphere outside the GWS disturbance. Changes in the belt/zone structure of the planets are known to happen with time scales ranging from a few days to some months, apart from the longer scale seasonal cycle (Pérez-Hoyos et al., 2006c).

As a first approach to determine whether or not a given latitude has been affected by changes in the overall vertical structure among the 18 dates used in this work, we decided to use the ratio between methane and continuum observations (last column on figures 1 and 2). For a given vertical cloud structure, the ratio between those observations is related to the effective column of methane, which, in the end, is related to the vertical distribution of aerosols. The correlation (or lack of) between methane and continuum observations has been previously discussed, for example, by Roman et al. (2013). This ratio proved to be a valuable tool in order to disentangle the apparent changes from the real ones. For this work, we have computed the ratio of the intermediate methane band filter MT2 to the adjacent continuum CB2. These filters are so close in the spectral range (some $25 \mathrm{~nm}$ in central 
passband wavelength) that any change in their reflectivity values can be attributed to differences in methane absorption inside and outside the band. Higher values of the ratio represent high and thick hazes, such as those in the Equatorial Zone, while belts displaying thinner and lower haze tops, have also lower values for the ratio, due to the increased methane absorption due to a longer atmospheric free path. While using the MT3/CB2 ratio would have maximized the methane to continuum difference, the MT3 images were usually acquired with lower spatial resolution (binned for a faster acquisition) and, moreover, limb-darkening behavior changes too much to be compensated with those filters even relatively close to the central meridian.

\subsection{Latitude selection}

In this study we use the MT2/CB2 ratio as a first approach to the overall distribution of aerosols in the upper troposphere and a similar value of the ratio is assumed to imply a very similar distribution of such aerosols. It could be argued that an adequate change in particle phase function or single scattering albedo could mask a change in the vertical distribution of particles. However, we find that the zonal mean of the ratio is remarkably stable for most latitudes (see figure 3) and that the discrepancies are found at the latitude band identified by morphological and dynamical analyses (SánchezLavega et al., 2012; Sayanagi et al., 2013).

In order to determine the stability of a given latitude, we compute the standard deviation $\sigma$ between the zonal means of all dates for the selected location. Extreme phase angles $\left(\alpha<20^{\circ}\right.$ and $\left.\alpha>100^{\circ}\right)$ sometimes divert from the rest of dates and were removed from this comparison. The differences for these observations are due to the amplitude of the forward and backscattering 
peaks of the planetary phase function. In the intermediate range, however, the phase function is apparently flat enough to allow comparisons. As shown in table 1, extreme phase angles are always accompanied by observations at intermediate phase angles in less than a week. This means that only physical changes in time-scales smaller than a week will be overseen with this method. The standard deviation as a function of latitude can be seen in figure 3. It has a constant value of $\sigma \sim 0.012 \pm 0.002$ except in the region between $20^{\circ} \mathrm{N}$ and $40^{\circ} \mathrm{N}$, where it grows strongly. Additionally, since there were no high phase angle data between $15^{\circ} \mathrm{N}$ and $20^{\circ} \mathrm{N}$, we decided to omit all latitudes between $15^{\circ} \mathrm{N}$ and $40^{\circ} \mathrm{N}$ from our analysis and separate the observations into two distinct groups: the Equatorial Zone with latitudes less than $15^{\circ} \mathrm{N}$ and extended phase coverage, and a mid-latitude regions with latitudes between $40^{\circ} \mathrm{N}$ and $70^{\circ} \mathrm{N}$ and more limited phase coverage.

The Equatorial Zone (EZ, latitudes less than $15^{\circ} \mathrm{N}$ ) has the highest haze tops in the whole planet, as it has already been noted in the past (Pérez-Hoyos and Sánchez-Lavega, 2006a). This region is very interesting dynamically, not only because of the strong vertical wind shear revealed by Hubble Space Telescope data (Sánchez-Lavega et al., 2003) but also because of a strong jet detected at high altitudes through Cassini ISS data, e.g. García-Melendo et al. (2010). Tomasko and Doose (1984) investigated corresponding southern latitudes roughly one Saturnian year earlier and thus this is a very interesting place for a direct comparison with their results.

In contrast, the mid-latitude zone is surrounded by two darker belts; the poleward belt borders the subpolar latitudes, where high altitude hazes may exhibit different particle properties (Karkoschka and Tomasko, 2005). 
At mid-latitudes the hazes are known to change in reflectivity from summer to winter, revealing changes in the overlying vertical cloud structure (Karkoschka and Tomasko, 2005). The Cassini observations used in the present study represent a snapshot of the northern hemisphere at spring equinox.

The initial latitude grid for modelling was arbitrarily sampled every $0.5^{\circ}$ in latitude. For every date and latitude, we selected 5 points uniformly distributed along the longitudes with illumination and observation angles below $85^{\circ}$ at each wavelength to be fit by our radiative transfer code.

\section{Radiative transfer model}

\subsection{Model atmosphere}

As in our previous works (Pérez-Hoyos et al., 2005; Sanz-Requena et al., 2012), Saturn's atmosphere was initially considered to be divided into three distinct layers of particles. Figure 4 shows a simple scheme of this distribution. The top layer is located in the stratosphere and its bottom level, $\mathrm{P}_{2}$, is arbitrarily fixed at 100 mbar, a mean value for the tropopause level. Initial tests showed no strong sensitivity to the $\mathrm{P}_{2}$ value, except for its effect on the total optical thickness of the layer. Particles are assumed to be spherical with their radius being one of the free parameters of the model. They are therefore described using a Mie phase function with the parameters presented in Table 2. In this table, $m_{r}$ and $m_{i}$ are, respectively, the real and imaginary parts of the refractive index, assumed to be that of the ammonia ice (Martonchik et al., 1984) but again with little sensitivity for this assumption. The size distribution is log-normal (Hansen and Travis, 
1974) with mean radius $r_{g}$ and variance $\sigma_{g}$. Particle number density $N_{1}$ is considered constant between $P_{1}$ and $P_{2}=100 \mathrm{mbar}$ (i.e. with a mixing ratio increasing with height), but it decays exponentially above $P_{1}$ with a scale height $H_{1}$ to be retrieved by the model.

The second, intermediate layer is where most of the photons at the wavelengths used here are scattered to the observer. This layer was assumed to be located from somewhere around the tropopause $\left(P_{3}\right)$ down to a place between the radiative-convective level and the expected condensation cloud at 1.4bar, here fixed to be at $P_{4}=1 b a r$. Most of the filters used here are not sensitive to this depth, and thus reaching the bottom of the haze and thus the data are only weakly sensitive to altitude though strongly sensitive to total optical thickness of the layer. Please note that it is certainly possible that both hazes overlap. NEMESIS is able to manage overlapping particle layers, so no constraints have been imposed on the limits of the hazes.

The phase function used for this layer is a double Henyey-Greenstein parameterized phase function (Henyey and Greenstein, 1941) as in Tomasko and Doose (1984). This phase function can be written as:

$$
P(\theta)=f \cdot \frac{1-g_{1}^{2}}{\left[1+g_{1}^{2}-2 g_{1} \cos (\theta)\right]^{3 / 2}}+(1-f) \cdot \frac{1-g_{2}^{2}}{\left[1+g_{2}^{2}-2 g_{2} \cos (\theta)\right]^{3 / 2}}
$$

In this expression, $\theta$ is the scattering angle and $P(\theta)$ the phase function. This two-term Henyey-Greenstein phase function (2HG) is composed of two terms with two asymmetry parameters $g_{1}$ and $g_{2}$. The first one is usually positive and therefore represents the forward scattering. The second one is, instead, used to describe the backward scattering, while parameter $f$ accounts for the relative weighting between them. In the radiative transfer equation 
written for a plane-parallel atmosphere (Liou, 1992; Sánchez-Lavega, 2011) the normalized phase function is multiplied by the single scattering albedo $\varpi_{0}$. This parameter is the ratio of the particle absorption relative to the particle scattering and perfect absorbers have therefore $\varpi_{0}=0.0$ and perfect scatterers $\varpi_{0}=1.0$. Highly reflective particles at longer wavelengths are commonly assumed (Pérez-Hoyos et al., 2005) since no evidence of particle absorption has been reported. It must be noted that in order to translate to particle number density to optical thickness, a particle cross section is required. We assumed a particle radius of $1 \mu \mathrm{m}$ with geometric cross section of $\pi \times 10^{-8} \mathrm{~cm}^{2}$ for the tropospheric particles at all latitudes. Retrievals are sensitive to optical thickness or extinction, e.g. particle number density is only as reliable as our estimation of the cross section is. $N_{2}$ could be off if the actual particle cross section is different from the value assumed here, but the product of both $\left(\tau_{\text {trop }}\right)$ can still be robustly retrieved.

As in the previous case, this haze is delimited by top $P_{3}$ and bottom $P_{4}$ pressures, with the latter fixed to $P_{4}=1 b a r$. Again, the particle number density $N_{2}$ is free but constant in this interval and set to decay with a free scale height $\mathrm{H}_{2}$, independent of that of the stratospheric haze.

Finally, a cloud deck was also placed from the bottom of the previous layer $\left(P_{5}=1 b a r\right)$ to the expected ammonia condensation level $\left(P_{6}=1.4 b a r\right)$. Although initial tests did not show a significant sensitivity, the particle number density $N_{3}$ was used as the main free parameter of this cloud. We assumed an isotropic phase function with a fixed single scattering albedo of $\varpi_{0}=0.995$ (Pérez-Hoyos et al., 2005), and the cross section of $10 \mu \mathrm{m}$ particles $\left(\pi \times 10^{-6} \mathrm{~cm}^{2}\right)$, see West et al. (2009) and references therein. The 
assumption of an isotropic phase function has no influence on the results, as we tested running the forward model with alternative phase functions (double Henyey-Greenstein and Mie phase function with arbitrary values).

In order to complete the vertical cloud structure model the gas behavior also has to be defined. Our atmosphere is simply described by a mixture of $\mathrm{H}_{2}$ and $\mathrm{He}$ that produces Rayleigh scattering, although this effect is only substantial at the shortest wavelengths covered here (BL1), where $\tau_{\text {Rayleigh }}$ $=0.559$ at 1 bar. The other active species is methane, whose volume mixing ratio has been taken as $4.7 \times 10^{-3}$, relative to $\mathrm{H}_{2}$ (Fletcher et al. , 2009). In order to calculate the absorption coefficient of methane we used the coefficients published by Karkoschka and Tomasko (2010). The pressure-temperature profile has little influence on the calculated absorption coefficient and that from Lindal et al. (1985) was used.

\subsection{Radiative transfer code}

The radiative transfer code used for this work is NEMESIS. NEMESIS stands from Non-linear optimal Estimator for MultivariatE spectral analySIS and was developed by the Oxford team (Irwin et al., 2008). It uses an optimal estimator technique (Rodgers, 2000) in order to perform multivariate retrievals of atmospheric properties from thermal or reflected radiation from the planets. It has become by now a standard code for radiative transfer in planetary atmospheres. Here, the code is used essentially as a forward model. The correlated-k method version of NEMESIS was used, based in the methane absorption coefficients by Karkoschka and Tomasko (2010). NEMESIS assumes a plane-parallel atmosphere for scattering and it uses a doubling/adding scheme (Hansen and Travis, 1974). 
Table 2 summarizes the atmospheric model parameters and the range of their values used to start the radiative transfer model. Many of these parameters, such as particle size or phase function parameters, cannot be treated as free parameters in the version of NEMESIS code used here, so we opted for a different approach that will be explained in the next section. This limitation has been removed in more recent versions of the code (Irwin et al., 2015).

\subsection{Optimization and retrieval}

Table 2 shows the 12 free parameters that should be constrained for every $0.5^{\circ}$ in latitude by comparing the model results with the observations. This is a huge computational effort that cannot be solved by a brute force approach in a reasonable amount of time. NEMESIS could constrain efficiently some of the parameters (e.g. those referring the vertical distribution of particles) but this would leave others aside, in particular those regarding the particle phase function. This would create a hierarchy between free parameters and, after several initial runs, we realized that all parameters should be treated equally and fitted at the same time. Thus NEMESIS is used here as a forward model, rather than as an optimization suite.

Finding the best-fitting model for each case was prioritized over fully sampling the 12-dimensional free parameter space to completely describe the a posteriori space. For doing so, a simplex method (Nelder \& Mead, 1965) was used to minimize the error function, described as the mean quadratic deviation $\chi^{2}$ between data and models, as in many of our previous works (e.g. Pérez-Hoyos et al. (2012)). This is a reduced $\chi^{2}$, an average of all the filters and positions over the disk being modelled. The simplex method iterates 
over a $N$-dimensional space (the logarithm of each free parameter, to avoid negative values and scale problems) and it evaluates the function in $N+1$ points (a simplex), substituting one of the vertices in each iteration. Iteration is terminated when certain conditions are met. In our case the termination condition was that $\chi_{i}^{2}-\chi_{i+1}^{2}<10^{-3}$, i.e. that the mean quadratic deviation did not get better than a thousandth with respect to previous iteration. This resulted in some 500 iterations for each case.

For every latitude, 10 initial models were randomly determined and were let to evolve until convergence, thus resulting in some 5,000 models ran for every latitude. Note that no termination condition over the absolute value of $\chi^{2}$ was imposed, so the value assumed for relative error $\sigma$ was of little importance. The model with minimum $\chi^{2}$ was selected as the best-fitting case. We show in Table 2 the initial range for initialization of every fitting run.

\subsection{Sensitivity to model parameters}

The large number of models run allows us to perform a statistical analysis for the sensitivity of the model to the parameters. Since the simplex method iterates many more times closer to the optimal solution, the most frequent values of a given parameter are also those with lower values of $\chi^{2}$. We can increase this effect by weighting the frequency by the probability of the model of being correct. For the most simple case of a problem with a single degree of freedom, the probability of a given model of being true (as opposed to being similar to observations just by chance) can be defined following de Kleer et al. (2015), where $\ln P \propto-\chi^{2} / 2$. Since we have used the reduced $\chi^{2}$ for model evaluation, we will explore model sensitivity scaling the frequency of a given 
parameter by $e^{-\chi^{2} / 2}$. This provides a sampling of the free parameter space and good information on how the best solutions are distributed in it. Figure 5 shows an example of the exploration of the free parameter space. The best sensitivity is shown as sharp maximums in the histograms (e.g. as in $\mathrm{N}_{2}, \mathrm{P}_{3}$ or $\varpi_{0}$ ), while low sensitivity is demonstrated by flat or multi-modal histograms (e.g. as in scale heights $H_{1}$ and $H_{2}$ or $N_{3}$ ). The sensitivity is good for $f$ ang $g_{1}$ parameters of the phase function, but $g_{2}$ is shown to have a broad distribution and therefore our sensitivity to this parameter is going to be lower.

Although the number of models explored and their distribution in the free parameter space is not optimal for this purpose, we can try to map the degeneracies between parameters as shown in figure 6 , where some combinations of free parameters are displayed. There are some obvious degeneracies in the distribution of tropospheric particles, such as $N_{2}$ and $P_{3}$ or $N_{2}$ and $\mathrm{H}_{2}$ which show that an adequate change in both parameters simultaneously could provide a model marginally as good as the best one. In the case of the phase function parameters, $g_{2}$ is in particular harder to determine because the backscattering can be produced by the combination of other parameters and thus it is not well constrained by our method. However, these results must be taken with caution and other methods which are statistically better founded should be used (de Kleer et al., 2015) at the cost of a much higher computational effort.

In order to determine the error bars for every parameter retrieval, we used a similar approach as in Pérez-Hoyos et al. (2012), rather than being based on the average statistics shown in figure 5 and 6 . Once we have determined the 
optimal model within the framework described in the previous paragraphs, we scan the free parameter space at one dimension each time, thus analysing the one-dimensional dependence of $\chi^{2}$ with every parameter. We arbitrarily defined error bars when the value of $\chi^{2}$ is twice that of the minimum. This relative criterion avoided having lower error bars when error is closer to the absolute limit (typically $\chi^{2}=1$ or something similar). Still, the error bars retrieved in this way were not very different from those estimated from the width of histograms described in the previous paragraph and are in good agreement with the dispersion of values for similar latitudes, as it will be shown in section 4 .

\section{Results}

\subsection{General results}

Using the techniques described in the preceding section, we are able to fit simultaneously the center to limb scans at the four filters for the 18 cases in Table 1 . The value of the reduced $\chi^{2}$ for all latitudes is shown in figure 7. Most fits are around $\chi^{2}=1$, which is acceptable. Some example fits are shown in figures 8 and 9 for two selected latitudes not particularly good or bad.

As it will be discussed later, there is a clear dependence of most parameters with latitude, both in their vertical distribution and in their scattering properties (phase function). In particular, there is a clear difference between the Equatorial atmosphere and those latitudes above the band disturbed by the storm. It is even possible to distinguish between the purely mid-latitudes (up to some $55^{\circ} \mathrm{N}$ ) and the region polewards of this. However, there are less 
data for these sub-polar latitudes and observation/illumination angles are more restricted, which results in a higher dispersion of the results. The sub-polar and polar latitudes will be analysed elsewhere using better suited observations.

\subsection{Uncertainties and limitations}

The error bars are substantially different depending on the free parameter, and they are in good agreement with the dispersion of the values in the same latitude region. Results concerning the vertical distribution of tropospheric aerosols are the best-constrained ones with relative errors around $5 \%$ for $\mathrm{P}_{3}$ and $20 \%$ for $\mathrm{N}_{2}$ and $\mathrm{H}_{2}$. The stratospheric haze, instead, yields relative errors that exceed $100 \%$ for most latitudes and free parameters and thus only help, in the best case, to provide upper/lower limits. Only the radius of stratospheric particles can be constrained occasionally with error bars around $10 \%$. The parameters of the phase function of the tropospheric particles, which are the main goal of this paper, can be commonly retrieved with uncertainties lower than $20 \%$, although the dispersion of results for similar latitudes are usually below that benchmark. The single scattering albedo of the particles is a very sensitive parameter that is usually determined better than $10 \%$.

As seen in figures 8 and 9, most of the model deviation from data is contributed by MT2 simulations at high emission angles. The points with emission angles higher than $70^{\circ}$ concentrate most of the error and are responsible for the increased values of $\chi^{2}$. However, this only happens with at MT2 wavelengths and thus can hardly be attributed to a problem with the plane parallel approximation. In order to understand the origin of such 
discrepancies, we ran three new retrievals for a few latitudes starting from the best-fitting model already obtained. For these calculations we used three different approaches: (1) We fit MT2 data at all phase angles simultaneously but regardless of the results at the other wavelengths; (2) We fit only low phase angles, but at all filters simultaneously; and (3) Same as (2) but for an intermediate phase angle $\alpha=80^{\circ}$.

The first approach tends to reduce the stratospheric haze optical thickness, or even remove it completely $\left(N_{1} \rightarrow 0\right)$. For mid-latitudes with initially less particles in the levels above 100 mbar, the model tends to change the scale height of the tropospheric haze $H_{2}$. This way, $\chi^{2}(M T 2)$ is reduced in a factor of 2 at the cost of increasing the error at other wavelengths, in particular at MT3, where it can be increased by factor of 10 .

When we try to fit a single phase angle, either at low or intermediate phase angles, but all wavelengths at the same time, we also have better fits than the ones used here. Changes around $25 \%$ in $N_{2}$ and $P_{3}$ are usually enough to reduce the reduced $\chi^{2}$ in a factor of 1.5. While $f$ and $g_{1}$ are esentially the same, backscattering parameter $g_{2}$ is also altered by about $10 \%$ when studying a low phase angle alone. However, we were not able to find any better model at low and high phase angles simultaneously.

All in all, our model works well at low and intermediate emission angles but it is just fair for emission angles above $70^{\circ}$, particularly at MT2. Our analysis above implies that the description of the particle distribution at $10 \mathrm{~s}$ of mbar, around the top of the tropospheric haze, could be improved with a more sophisticated distribution or with a better vertical resolution. However, it must be noted that results for phase function parameters are still robust 
and within the retrieved error bars.

\subsection{Phase function}

As shown in figure 10, the results for the phase function show at least two distinct regions of the planet. While the weighting between the forward and the backward backscattering is almost the same all over the planet, the backward and forward parameters change abruptly somewhere between the Equatorial Zone and the mid-latitudes.

Considering all latitudes, the average value for all wavelengths of $f$ is $0.85 \pm 0.06$. This is slightly smaller in the $\mathrm{EZ}(f=0.79 \pm 0.04)$ than at midlatitudes $(f=0.88 \pm 0.04)$, almost within retrieval error bars. Parameter $g_{1}$ changes from $g_{1}=0.75 \pm 0.09$ for latitudes close to the Equator to $g_{1}=$ $0.83 \pm 0.04$ for latitudes poleward of the region disturbed by the 2010 giant storm (GWS). Parameter $g_{2}$ is more stable, ranging from $g_{2}=-0.33 \pm 0.05$ at the EZ to $g_{2}=-0.31 \pm 0.07$. The global averages for both parameters are $g_{1}=0.81 \pm 0.07$ and $g_{2}=-0.31 \pm 0.07$.

We tried to get better fits by leaving free the parameters of the $2 \mathrm{HG}$ phase function for the blue wavelengths. However, these observations are very sensitive to the single scattering albedo and they do not provide any significant changes in $f, g_{1}$ or $g_{2}$. However, the single scattering albedo $\varpi_{0}(B L 1)$ can be easily constrained and provides values clearly below those used at NIR wavelengths. Figure 11 shows its value as a function of latitude. Once again, there is a clear distinction between the Equatorial aerosols and those located at higher latitudes. Mean values are $\varpi_{0}(B L 1)=0.94 \pm 0.02$, but this is $\varpi_{0}(B L 1)=0.92 \pm 0.01$ for $\mathrm{EZ}$ and $\varpi_{0}(B L 1)=0.95 \pm 0.01$ for the rest of latitudes. 


\subsection{Spatial distribution}

The main results for the parameters regarding the stratospheric haze are shown in figure 12. However, our model is not very sensitive to these parameters, in particular because the total integrated optical thickness is low, with average values of $\tau_{\text {str }}=0.01 \pm 0.01$ at $890 \mathrm{~nm}$. However, there is some tendency in the results with the Equatorial Zone displaying higher hazes, although average values of particle density do not differ substantially. Particle radius seems also to be slightly higher in the low latitudes than polewards of the region disturbed by the storm.

The model excels in fitting the parameters of the tropospheric haze, as shown in figure 13. In particular, it is very sensitive to its top pressure level, with the smaller error bars in this study, as it has been already pointed out. The tropospheric haze top seems to be substantially above the tropopause level for the EZ, which in any case is not well defined at these latitudes as analysed from radio occultations (Schinder et al., 2011). At mid-latitudes, it reaches the 60 mbar level around the latitudes where the mid-latitude jet is located (García-Melendo et al., 2011).

Particle number density is $N_{2}=5 \pm 1 \mathrm{part} / \mathrm{cm}^{3}$ in the EZ and somewhat higher at mid-latitudes $\left(N_{2}=7 \pm 1 \mathrm{part} / \mathrm{cm}^{3}\right)$, excluding those above $60^{\circ}$, which peak at $N_{2}=10 \pm 5$ part $/ \mathrm{cm}^{3}$ but with a high dispersion, possibly due to the limited coverage of the scattering angles at those latitudes which prevents observing the whole limb-darkening curve from an equatorial orbit.

The haze is assumed to decay exponentially above such level with scale heights lower than those of the gas in Saturn. In the EZ, the scale height is $H_{g} / H_{p}=10 \pm 5$, while it is about $H_{g} / H_{p}=5 \pm 2$ for the rest of latitudes. Since 
the gas scale height in Saturn is about $35 \mathrm{~km}$ at the 100 mbar pressure level, this implies a scale height for the haze of $\sim 3.5 \mathrm{~km}$ and $\sim 7 \mathrm{~km}$, respectively.

It is possible to integrate the retrieved particle number density from the top of the atmosphere to retrieve the total optical thickness for the stratospheric (at a reference wavelength) and tropospheric (independent of wavelength by construction) hazes, as shown in figure 14 . This obviously resembles the distribution of particle number density but it is very useful for comparison in previous works (see next section). The stratospheric haze has a total optical thickness of $\tau_{\text {str }}=0.03 \pm 0.02$ at $890 \mathrm{~nm}$ in the EZ. In the region poleward of the storm, the upper limit seems to be 0.1 , except for the most polar latitudes where the retrievals are much noisier. The total optical thickness of the tropospheric haze is remarkably constant with latitude, with averages of $\tau_{\text {trop }}=7 \pm 2$ for both the Equatorial and mid-latitudes. Values and uncertainties, again, are higher in the polar latitudes, above $60^{\circ} \mathrm{N}$.

A graphical representation of the vertical distribution of the tropospheric particles can be found in figure 15. In general terms, higher haze tops are correlated with lower particle scale heights, i.e. with hazes that end more abruptly. The latitudes with lower haze tops, conversely, are more extended with greater scale heights. Latitudes above $60^{\circ}$ are the extreme case, although it has already been noted, the retrieved values are substantially noisier and thus less reliable than those at other latitudes.

Finally, little or no information can be obtained about the latitudinal distribution of particles at deeper atmospheric levels. There is only a lower limit to the optical thickness of the bottom cloud $\left(\tau_{\text {cloud }} \geq 10\right)$. 


\section{Discussion}

\subsection{Tropospheric particle phase function}

The work by Tomasko and Doose (1984) is the only determination of the phase function for the case of Saturn that can be used to compare our results. This comparison is presented in table 3, where we show our averaged values for the Equatorial Zone $\left(0^{\circ}-15^{\circ} \mathrm{N}\right)$, the mid- and sub-polar latitudes $\left(45^{\circ}-70^{\circ} \mathrm{N}\right)$ and the global means $\left(0^{\circ}-70^{\circ} \mathrm{N}\right)$. Focusing on the EZ values, Tomasko and Doose (1984) results are within our retrieved error bars for $f$ and backscattering parameter $g_{2}$, while there is a substantial difference in the forward scattering parameter $g_{1}$. Thus, current results require a more forward scattering particle phase function than that observed in the late 1970s. This is quite reasonable, since there is no reason why we should expect a perfect match in mean particle sizes or shapes after one Saturn's year, considering also that we are working at different latitudes and wavelengths.

Although more limited in phase angle coverage, West et al. (1983) worked with observations of the Equatorial Zone obtained with the Voyager 2 photopolarimeter from $12^{\circ}$ to $68^{\circ}$ phase angles. Their resulting values of the Henyey-Greenstein differ from those presented here in the forward scattering $g_{1}$, which is lower in their work $(0.54 \pm 0.11$ vs $0.75 \pm 0.09)$ and $g_{2}(-0.47 \pm 0.08$ vs $-0.33 \pm 0.05)$, somewhat bigger in absolute value. The weighting parameter $f$ is also bigger $(0.87 \pm 0.03$ vs $0.79 \pm 0.04)$. In any case, all parameters are almost within respective error bars, which gives a marginal agreement between both analysis.

Although the $2 \mathrm{HG}$ phase function is a very useful parameterization of the phase function, it provides little insight into the underlying physics. For this 
reason, it is very useful to compare the results for the $2 \mathrm{HG}$ phase function with a Mie phase function, which assumes sphericity of the particles. However, polarization measurements at Jupiter using Pioneer IPP instrument discarded spherical particles as candidates (Smith \& Tomasko, 1984) and it is very likely that the same will be true for Saturn. As discussed for example in West et al. (2009), ice crystals expected at this atmospheric level cannot be modelled using spherical scatterers. Non-spherical particles in the form of aggregates have been proposed to explain observations of Jupiter's polar haze (West and Smith, 1991; Zhang et al., 2013) and could be ubiquituous in the upper atmospheres of the giant planets.

It is however possible to use spheroids as a simple approximation to the non-sphericity. With this purpose, we computed the phase function of spheroids with different mean sizes and aspect ratios. The optical properties were obtained from look up tables provided by Dubovik et al. (2006). These use T-matrix calculations for smaller particles combined with ray tracing for the larger particles. The tables have scattering properties at 25 different aspect ratios between 0.335 and 3.0; 22 real parts of refractive index between 1.29 and 1.70; 15 imaginary parts of refractive index between 0.0005 and 0.5 and 41 narrow radius bins with size parameters from 0.02 to 626 . For imaginary parts of refractive index below 0.0005 , there is very little change in optical properties (since at these values, there is close to no absorption) so this value is appropriate for all values below 0.0005 . From the simulation results, it is clear that the forward scattering is the best indication of the particle size, while the backscattering is more influenced by the shape or aspect ratio. 
The phase functions for the Equatorial Zone and the mid-latitudes are shown in figure 16. At the equator, scattering angles from $\sim 20^{\circ}$ to $\sim 170^{\circ}$ are covered and thus we have a excellent sampling of the phase function. The forward peak at the EZ is in good agreement with ellipsoids of mean radius $1 \pm 0.2 \mu \mathrm{m}$, but they do not reproduce well the intermediate scattering angles even in the best-fitting case of aspect ratio $<0.6$ and the fit is marginal at the best case. We did not find any better fit for EZ particles using ellipsoids. Yet, ammonia ice crystals have been proposed (West et al., 2009; Tomasko and Doose, 1984) to be responsible of the observed phase function. We compare in figure 16 with laboratory data of $10 \mu \mathrm{m}$ ammonia ice crystals retrieved by Pope et al. (1992). The match is very good and provides a good indication of the expected size and nature of the tropospheric particles at the Equatorial Zone.

In the case of non-equatorial particles, ammonia ice is not in good agreement with the retrieved phase function at intermediate scattering angles. Ellipsoids, instead, yield an excellent fit again with mean radius $1 \pm 0.2 \mu \mathrm{m}$ and aspect ratio $<0.6$. There is a marginal solution with smaller particles of mean radius $0.25 \pm 0.05$ and very elongated (aspect ratio $>0.7$ ). This second solution, however, clearly misses the scattering at phase angles greater than $100^{\circ}$, so it is not favoured by our results. Such dichotomy would be broken if we had high phase angle observations for the mid-latitudes of Saturn. In order to understand how this affected our understanding of the phase function at these latitudes, we tried to fit the retrieved values of the phase function at the observed scattering angles with a different double Henyey-Greenstein function. It is possible to find another solution consistent at the observed 
angles using backscattering parameter $g_{2}<-0.9$. This, however, would result in a extremely strong increase of the phase function at low phase angles which implies a very intense opposition surge that has not been reported in Saturn's atmosphere, even though it has been observed in the rings.

A very interesting point is whether or not Equatorial particles can be said to differ substantially from the particles found at other latitudes, from the data and retrievals shown in this work. While we have some clues pointing in that direction (e.g. changes in particle phase function parameters), there is still a chance that particles at all latitudes are the same since results are marginally consistent with each other. Results are robust for EZ particles, but the lack of observations at the forward peak of the phase function makes results for the other latitudes less reliable. What we see is compatible with $1 \mu \mathrm{m}$ ellipsoids moderately elongated, but it could be certainly the case that ammonia ice crystals were also valid for the mid-latitudes.

\subsection{Particle distribution}

This work is the only attempt to date to reproduce the reflectivity of Saturn at a wide range of phase angles simultaneously since 1979. However, the results we retrieve from the radiative transfer analysis presented so far do not differ from our previous understanding of Saturn's atmosphere. Most works (Karkoschka and Tomasko, 2005; Pérez-Hoyos et al., 2005; Temma et al., 2005; Roman et al., 2013) assume a distribution of aerosols in two levels, as we do in this work. Even though our assumptions may differ from those adopted by others, this basic agreement in the description of the atmosphere allows comparing many atmospheric parameters.

Regarding the overall distribution of tropospheric particles, the Equato- 
rial Zone is in all works the region of the atmosphere displaying higher and thickes hazes, as is for example summarized in figure 1 of Pérez-Hoyos and Sánchez-Lavega (2006a). The Equatorial Zone haze top retrieved here is also located substantially above the tropopause, resembling the results by Roman et al. (2013). While the optical thickness of the Equatorial tropospheric haze is 2-4 times lower than the values retrieved from observations in the 1990s (Pérez-Hoyos and Sánchez-Lavega, 2006a), the values found here are very similar to those found in figure 4 from Roman et al. (2013).

At other latitudes, the analogy with the Southern Hemisphere investigated by Roman et al. (2013) still holds in both the optical thickness and top pressure, although they also modelled the bottom pressure, here assumed to be fixed at $P_{4}=1$ bar. We find a significant difference for example with Pérez-Hoyos et al. (2005), where the optical thickness at the EZ is 5-10 times higher than at the middle latitudes. Something similar happens with Karkoschka and Tomasko (2005), where they found a clear increase in the optical thickness of the tropospheric haze towards lower latitudes. Karkoschka and Tomasko (2005) also have tropospheric haze tops at lower levels for most years. It must be noted that these works were based on observations of the Southern Hemisphere (Pérez-Hoyos et al., 2005) at a different epoch, so this can be either a seasonal effect or a difference in the modelling approach.

Most of the works cited above used a hard boundary for the tropospheric haze, but in this work we found that agreement between MT2 and MT3 was better using an additional free parameter describing the particle scale height. The work by Tomasko and Doose (1984) also gave a particle scale height at the equatorial latitudes of $H_{g} / H_{p}=3$. This value is within the error bars 
of our results for the mid-latitudes, but our equatorial hazes in the current epoch have a much more pronounced slope and decay more rapidly (i.e. their scale height is some three times lower than in the Pioneer epoch).

Overall, the retrieved values of optical thickness retrieved for the hazes are around the consensus established for Saturn before the Cassini spacecraft were in orbit. As already shown in section 6 , total optical thickness for the tropospheric haze is found to be relatively constant with latitude with a mean value of $\tau_{\text {trop }} \sim 7$. This could look in contradiction with the commonly assumed paradigm that Equatorial clouds are substantially thicker than at other latitudes. This is based in the lack of thermal emission for example at $5.1 \mu \mathrm{m}$, see figures 7.18 and 7.19 in West et al. (2009). However, it must be noted that we are referring to opacity in the upper bar of the atmosphere and that our model is compatible with a substantial source of cloud opacity below that level.

The single scattering albedo at blue wavelengths retrieved here can be compared directly with the values by Pérez-Hoyos et al. (2005). While they cover a greater time span and a different filter selection, their values are clearly lower than ours. Most of they retrievals have $\varpi_{0}<0.9$, and our values are above that threshold for all latitudes. This could be related with the fixed phase function assumed in their work, here found to be somewhat different, which can compensate for the differences in the values of the single scattering albedo.

Finally, it is certainly very difficult to make any comparison regarding the parameters of the stratospheric haze, given the low sensitivity our analysis has shown to them. Low values of optical thickness and small particle 
sizes are in good agreement with most retrievals (Pérez-Hoyos et al., 2005; Karkoschka and Tomasko, 2005; Roman et al., 2013); however it, is no clear that an increase of particle number density towards the poles can be clearly concluded from the results shown here.

Overall, the particle distribution retrieved here is not far from the common picture described in previous works and differences are within retrieval uncertainties in most cases. Although there are many differences in the approach by many teams, it looks like the general distribution of particles around the tropopause level can be confidently retrieved from observations only at low phase angles, which is our most common source of information in the absence of spacecrafts orbiting the planet.

\subsection{Dynamical implications}

There is a very interesting point that connects the kind of analysis developed here and the atmospheric dynamics we are seeing in the atmosphere. The radiative transfer analysis is the only way we have to determine the vertical location of the features used to determine the flows in the atmosphere and the local meteorological phenomena. This implies the analysis of the actual features used for, say, wind tracking, but we can gain some insight by inspecting the contribution functions of each filter through the average atmosphere, shown in figure 17. From this figure we can learn that these contribution functions are aerosol dominated and therefore, they seem to sound relatively similar pressure levels in the atmosphere. Figure 17 only shows MT3 and CB2 contribution functions since they are the two extreme cases, commonly used for cloud tracking for regions with detected wind shear, as in García-Melendo et al. (2010, 2011). 
As noted above, particles at low latitudes have a local peak very high in the atmosphere and then the contribution function decreases faster than it does at the middle and sub-polar latitudes. This leaves more space in the atmosphere for the gas absorption to influence the contribution functions. Thus, the MT3 contribution function isolines in figure 17 are higher in the atmosphere and more concentrated, meaning that we are less prone to detect anything that could be located below the 200 mbar level, while they are potentially sensitive to levels as high as 10 mbar. By looking through the CB2 filter, instead, we could potentially detect anything down to the 1 bar level. It is not only about being able to see through the hazes but also about having actual atmospheric features at a given altitude level. Moreover, MT2 and MT3 are very sensitive to height changes of individual features near the tropopause while CB2 observations would be insensitive to this kind of differences. This could help us to understand the vertical wind shears detected not only in the EZ (Sánchez-Lavega et al., 2003; Vasavada et al., 2006) but also the smaller shears at the peak jets at other latitudes (GarcíaMelendo et al., 2011) or their lack of. It is also in close agreement with the idea that we could be sounding much higer levels in the EZ in the methane absorption bands than at the continuum and therefore witnessing dynamics located much higher in the atmosphere (García-Melendo et al., 2010).

\section{Conclusions}

In this work, we have analyzed a set of Cassini ISS observations covering blue wavelengths $(451 \mathrm{~nm})$, two methane bands (centered at 727 and 890 $\mathrm{nm})$ and intermediate continuum $(750 \mathrm{~nm})$ that cover a wide range of phase 
angles providing the first chance since 1979 Pioneer 11 analysis to perform a systematic analysis of the particle phase function. Given the wavelengths covered in this work, this phase function is restricted to the upper troposphere levels, which most strongly contribute to the reflected radiation observed in visual wavelengths.

We summarize below the most important points of this work and the most important conclusions that can be drawn from it:

- While the atmosphere was perturbed in 2010 by the uplift of the GWS, this perturbation was restricted to a latitude band between $15^{\circ}$ and $40^{\circ}$ and the rest of the northern hemisphere latitudes show no sign of substantial variation in the reflectivity at the considered filters.

- The observed reflectivity can be reproduced as a function of wavelength for all the latitudes of interest and emission angles below $80^{\circ}$, by using a relatively simple model for the vertical distribution of particles. Models at higher emission angles depart from observations at MT2 filter, which require fewer particles above the tropopause level.

- Equatorial and mid-latitude hazes have a strongly forward scattering phase function. The phase function a the equator is in good agreement with $10 \mu \mathrm{m}$ ammonia ice crystals, while uncertainties due to limited phase coverage and parameter degeneracy prevent strong constraints of the particle shapes and sizes at mid- and sub-polar latitudes. These particles may be similar to the equatorial particles, but they may also be consistent with $1 \mu \mathrm{m}$ ellipsoids with moderate aspect ratios.

- Global means are similar to previous values provided by Tomasko and 
Doose (1984) although there are some differences. Most notably, our models do not require different phase function parameters for blue wavelengths, which can be reproduced with the same parameters as in the near infrared wavelengths. The main difference in the Equatorial phase function is that Tomasko and Doose (1984) has lower values of the forward scattering parameter, beyond the retrieval uncertainties.

- The overall integrated optical thickness and haze top values for the tropospheric haze are in close agreement with previous results regarding different seasons of the Saturnian year.

- Analysis of the contribution function for the filters commonly used for cloud tracking and most extremely sounding (MT3 and CB2), show that both are aerosol dominated. However, MT3 sounding levels are restricted from $\sim 10$ mbar down to $\sim 200$ mbar, while CB2 is able to sound well past the 1 bar level. This puts into context the detection of vertical wind shears for the Equator and is consistent with two separate dynamical levels being seen at some latitudes.

Now that we are approaching the end of the Cassini mission in 2017, it would be useful that analysis like the present one here could be undertaken for other latitudes and time spans, although it should be noted that both phase angle coverage and stability of the atmosphere at the latitudes of interest must be present. 
800

801

\section{Acknowledgements}

We gratefully acknowledge the work of the Cassini ISS team that made the data available. This work was supported by the Spanish MICIIN project and AYA2012-36666 with FEDER support, Grupos Gobierno Vasco IT76513, and UPV/EHU UFI11/55. S.P.-H. acknowledges support from the Jose Castillejo Program funded by Ministerio de Educación, Cultura y Deporte, Programa Nacional de Movilidad de Recursos Humanos del Plan Nacional de I-D+i 2008-2011. This work has benefited from the comments of Dr. R. West and Dr. M. Roman during the review process. 


\section{References}

Dubovik, O., A. Sinyuk, T. Lapyonok, B.N. Holben, M. Mishchenko, P. Yang, T.F. Eck, H. Volten, O. Muñoz, B. Veihelmann, W.J. van der Zande, J.-F. Leon, M. Sorokin, and I. Slutsker, 2006: Application of spheroid models to account for aerosol particle nonsphericity in remote sensing of desert dust. J. Geophys. Res., 111, D11208.

Fischer, G. et al., 2011. A giant thunderstorm on Saturn. Nature 475, 7577.

Fletcher, L.N., G.S. Orton, N.A. Teanby, P.G.J. Irwin, G.L. Bjoraker, 2009. Methane and its isotopologues on Saturn from Cassini/CIRS observations. Icarus 199, pp. 351367.

Fletcher, L.N. et al., 2011. Thermal structure and dynamics of Saturn's northern springtime disturbance. Science 332, 14131417.

Fletcher, L.N., K.H. Baines, T.W. Momary, A.P. Showman, P.G.J. Irwin, G. S. Orton, M. Roos-Serote, and C. Merlet, 2011. Saturns tropospheric composition and clouds from Cassini/VIMS 4.65.1 m nightside spectroscopy. Icarus 214, 510-533.

García-Melendo, E., A. Sánchez-Lavega, J. Legarreta, S. Pérez-Hoyos and R. Hueso, 2010. A strong high altitude narrow jet detected at Saturn's equator. Geophysical Research Letters 37, L22204.

García-Melendo, E., S. Pérez-Hoyos, A. Sánchez-Lavega and R. Hueso, 2011. Saturn's zonal wind profile in 2004-2009 from Cassini ISS images and its long-term variability. Icarus 215, 62-74. 
Hansen, J.E., Travis, L.D., 1974. Light scattering in planetary atmospheres. Space Sci. Rev. 16, 527610.

Henyey, L.C., Greenstein, J.L., 1941. Diffuse radiation in the Galaxy. Astrophys. J. 93, 7083.

Hueso, R. J. Legarreta, J.F. Rojas, J. Peralta, S. Prez-Hoyos, T. del RoGaztelurrutia and A. Snchez-Lavega, 2010. The Planetary Laboratory for Image Analysis (PLIA). Advances in Space Research, 46, 1120-1138.

Irwin, P.G.J., N.A. Teanby, R. de Kok, L.N. Fletcher, C.J.A. Howett, C.C.C. Tsang, C.F. Wilson, S.B. Calcutt, C.A. Nixon, P.D. Parrish, 2008. The NEMESIS planetary atmosphere radiative transfer and retrieval tool. Journal of Quantitative Spectroscopy and Radiative Transfer 109, 1136-1150.

Irwin, P.G.J., L.N. Fletcher, P.L. Read, D. Tice, I. de Pater, G.S. Orton, N.A. Teanby and G.R. Davis, 2015. Spectral analysis of Uranus' 2014 bright storm with VLT/SINFONI. Icarus 264, 72-89.

Karkoschka, E., Tomasko, M.G., 2005. Saturn's vertical and latitudinal cloud structure 19912004 from HST imaging in 30 filters. Icarus 179, 195221.

Karkoschka, E., Tomasko, M.G., 2010. Methane absorption coefficients for the Jovian planets from laboratory, Huygens, and HST data. Icarus 205, 674694 .

de Kleer, K., S. Luszcz-Cook, I. de Pater, M. Adamkovics, H.B. Hammel, 2015. Clouds and aerosols in Uranus: radiative transfer modeling of spatially-resolved near-infrared Keck spectra. Icarus 256, 120-137. 
Lindal, G.F., Sweetnam, D.N., Eshleman, V.R., 1985. The atmosphere of Saturnan analysis of the Voyager radio occultation measurements. Astron. J. 90, 11361146

Liou, K.N., 1992. Radiation and Cloud Processes in the Atmosphere. Oxford Univ. Press, New York.

Martonchik, J.V., Orton, G.S., Appleby, J.F., 1984. Optical properties of NH3 ice from the far infrared to the near ultraviolet. Appl. Optics 23,541547

Nelder, J.A. \& R. Mead, 1965. A Simplex method for function minimization. The Computer Journal 7, 308-313.

Pérez-Hoyos, S., Sánchez-Lavega, A., French, R.G., Rojas, J.F., 2005. Saturn's cloud structure and temporal evolution from ten years of Hubble space telescope images (1994-2003). Icarus 176, 155174.

Pérez-Hoyos, S., Sánchez-Lavega, A., 2006a. On the vertical wind shear of Saturn's Equatorial jet at cloud level. Icarus 180, 161175.

Pérez-Hoyos, S., Sánchez-Lavega, A., 2006b. Solar flux in Saturn's atmosphere: Penetration and heating rates in the aerosol and cloud layers. Icarus 180, 368378.

Pérez-Hoyos, S., Sánchez-Lavega, A. and R. G. French 2006c. Short-term changes in the belt/zone structure of Saturns Southern Hemisphere (19962004), Astronomy \& Astrophysics, 460, 641-645. 
Pérez-Hoyos, S., Sanz-Requena, J.F., Barrado-Izagirre, N., Rojas, J.F., Sánchez-Lavega, A., 2012. The 2009-10 fade of Jupiter's South Equatorial Belt: Vertical cloud structure models and zonal winds from visible imaging. Icarus 217, 256271.

Pope, S.K., M.G. Tomasko, M.S. Williams et al., 1992. Clouds of ammonia ice: Laboratory measurements of single-scattering properties. Icarus 100, 203-220.

Porco, C.C. et al., 2004. Cassini Imaging Science: Instrument characteristics and anticipated scientific investigations at Saturn. Space Sci. Rev. 115, 363497.

del Río-Gaztelurrutia, T., Legarreta, J., Hueso, R., Pérez-Hoyos, S., SánchezLavega, A., 2010. A long-lived cyclone in Saturn's atmosphere: Observations and models. Icarus 209, 665681.

Rodgers, C.D., 2000. Inverse methods for atmospheric sounding. Theory and practice. World Scientific, London.

Roman, M.T., D. Banfield and P.J. Gierasch (2013). Saturn's cloud structure from Cassini ISS. Icarus 225, 93-110.

Sánchez-Lavega, A., 2011. An Introduction to Planetary Atmospheres. CRC Press, Boca Raton, FL, USA.

Sánchez-Lavega, A., Colas, F., Lecacheux, J., Laques, P., Miyazaki, I., Parker, D., 1991. The great white spot and disturbances in Saturn's Equatorial atmosphere during 1990. Nature 353, 397401. 
Sánchez Lavega, A., J. Lecacheux, F. Colas, P. Laques, 1993. Temporal behavior of cloud morphologies and motions in Saturns atmosphere, Journal of Geophysical Research, 98 (E10), 18857 - 18872.

Sánchez-Lavega, A., S. Pérez-Hoyos, J. F. Rojas, R. Hueso and R. G. French, 2003. A strong decrease in Saturn's equatorial jet at cloud level. Nature, 423, 623-625.

Sánchez-Lavega, A., Pérez-Hoyos, S., Hueso, R., 2004. Clouds in planetary atmospheres: A useful application of the ClausiusClapeyron equation. Am. J. Phys. 72, 767774. doi:10.1119/1.1645279.

Sánchez-Lavega, A., Hueso, R., Pérez-Hoyos, S., 2007. The three-dimensional structure of Saturn's Equatorial jet at cloud level. Icarus 187, 510519.

Sánchez-Lavega, A. et al., 2011. Deep winds beneath Saturn's upper clouds from a seasonal long-lived planetary-scale storm. Nature 475, 7174.

Sánchez-Lavega, A., T. del Río-Gaztelurrutia, M. Delcroix, J J. Legarreta, J. M. Gómez-Forrellad, R. Hueso, E. García-Melendo, S. Pérez-Hoyos, D. Barrado-Navascus, Jorge Lillo, 2012. Ground-based Observations of the Long-term Evolution and Dead of Saturn's 2010 Great White Spot, Icarus, 220, 561-576.

Sanz-Requena, J.F., S. Pérez-Hoyos, A. Sánchez-Lavega, T. del RíoGaztelurrutia, D. Barrado-Navascués,F. Colas, J. Lecacheux, D. Parker, 2012. Cloud structure of Saturn's 2010 storm from ground-based visual imaging. Icarus 219, 142-149. 
Sayanagi, K.M., U.A. Dyudina, S.P. Ewald, G. Fischer, A.P. Ingersoll, W.S. Kurth, G.D. Muro, C.C. Porco and R.A. West, 2013. Dynamics of Saturns great storm of 20102011 from Cassini ISS and RPWS. Icarus 223, 460-478.

Schinder, P. J., F. M. Flasar, E. A. Marouf, R. G. French, C. A. McGhee, A. J. Kliore, N. J. Rappaport, E. Barbinis, D. Fleischman, and A. Anabtawi. Saturn's equatorial oscillation: Evidence of descending termal structure from Cassini radio occultations, Geophys. Res. Lett. 38, L08205.

Smith, P.H. and M.G. Tomasko, 1984. Photometry and polarimetry of Jupiter at large phase angles. II. Polarimetry of the South Tropical Zone, South Equatorial Belt, and the Polar Regions from the Pioneer 10 and 11 missions. Icarus 58, 35-73.

Sromovsky, L.A., K.H. Baines and P.M. Fry, 2013. Saturns Great Storm of 2010-2011: Evidence for ammonia and water ices from analysis of VIMS spectra. Icarus 226, 402-418.

Temma, T., Chanover, N.J., Simon-Miller, A.A., Glenar, D.A., Hillman, J.J., Kuehn, D.M., 2005. Vertical structure modeling of Saturn's Equatorial region using high spectral resolution imaging. Icarus 175, 464489

Tomasko, M.G., Doose, L.R., 1984. Polarimetry and photometry of Saturn from pioneer 11: Observations and constraints on the distribution and properties of cloud and aerosol particles. Icarus 58, 134.

Tomasko, M.G., West, R.A., Orton, G.S., Tejfel, V.G., 1984. Cloud and aerosols in Saturns atmosphere. In: Gehrels, T., Matthews, M.S. (Eds.), Saturn. Univ. of Arizona Press, Tucson, AZ, pp. 150194. 
Tomasko, M.G. and L.R. Doose, 1985. Clouds and aerosols on Saturn. In: ESA The Atmospheres of Saturn and Titan p 53-61 (SEE N86-28849 19$88)$.

Van de Hulst, H.C., 1957. Ligh scattering by small particles. Courier Dover Publications, New York.

Vasavada, A.R. et al., 2006. Cassini imaging of Saturn: Southern hemisphere winds and vortices. J. Geophys. Res. 111, E05004.

West, R.A., 1983. Spatially resolved methane band photometry of Saturn: II. Cloud structure models at four latitudes. Icarus 53, 301309.

West, R.A., M. Sato, H. Hart, A.L. Lane, C.W. Hord, K.E. Simmons, L.W. Esposito, D.L. Coffeen, and R.B. Pomphrey, 1983. Photometry and polarimetry of Saturn at 2640 and $7500 \AA$. J. Geophys. Res., 88, 8679-8697.

West, R. A., and P. H. Smith 1991. Evidence for aggregate particles in the atmospheres of Titan and Jupiter. Icarus 90, 330-333.

West, R.A., Baines, K.H., Karkoschka, E., Snchez-Lavega, A., 2009. Clouds and aerosols in Saturns atmosphere. In: Dougherty, M.K., Esposito, L.W., Krimigis, S.M. (Eds.), Saturn from Cassini-Huygens. Springer, New York, pp. 113159.

West, Robert; Knowles, Benjamin; Birath, Emma; Charnoz, Sebastien; Di Nino, Daiana; Hedman, Matthew; Helfenstein, Paul; McEwen, Alfred; Perry, Jason; Porco, Carolyn; Salmon, Julien; Throop, Henry; Wilson, Daren, 2010. In-flight calibration of the Cassini imaging science sub-system cameras. Planetary and Space Science 58, 1475-1488. 
964 Zhang, X., R.A. West, D. Banfield and Y.L. Yung, 2013. Stratospheric 965 aerosols on Jupiter from Cassini observations. Icarus 226, 159-171. 
Table 1: Summary of the Cassini ISS observations.

\begin{tabular}{cccc}
\hline Date & $B$ & $B^{\prime}$ & $\alpha$ \\
\hline $2010-12-05$ & -0.02 & 7.21 & 80.2 \\
$2010-12-23$ & -0.02 & 7.46 & 60.8 \\
$2010-12-24$ & -0.03 & 7.48 & 71.2 \\
$2011-01-06$ & -0.07 & 7.66 & 113.3 \\
$2011-01-09$ & -0.07 & 7.71 & 146.9 \\
$2011-01-15$ & 0.26 & 7.80 & 78.0 \\
$2011-01-21$ & 0.31 & 7.88 & 95.4 \\
$2011-03-07$ & 0.37 & 8.52 & 84.8 \\
$2011-03-19$ & 0.17 & 8.69 & 157.7 \\
$2011-04-22$ & 0.29 & 9.16 & 57.6 \\
$2011-05-03$ & 0.37 & 9.31 & 86.3 \\
$2011-06-14$ & 0.38 & 9.90 & 94.8 \\
$2011-07-14$ & 0.10 & 10.31 & 20.5 \\
$2011-08-03$ & 0.03 & 10.58 & 12.1 \\
$2011-08-06$ & 0.16 & 10.63 & 28.2 \\
$2011-09-06$ & 0.30 & 11.05 & 53.6 \\
$2011-11-30$ & 0.20 & 12.18 & 41.8 \\
$2011-12-31$ & -0.79 & 12.58 & 79.4 \\
\hline$R .03$ & \\
\hline
\end{tabular}

$B$ : sub-spacecraft planetocentric latitude $B^{\prime}$ : sub-solar planetocentric latitude $\alpha$ : phase angle 
Table 2: Model atmosphere parameters.

\begin{tabular}{|c|c|c|c|}
\hline Layer & Parameter & Type & Value \\
\hline \multirow{8}{*}{ Stratospheric Haze } & $P_{1}$ & Free & 0.1-100 mbar \\
\hline & $P_{2}$ & Fixed & 100 mbar \\
\hline & $N_{1}$ & Free & $10^{-3}-10^{1} \mathrm{part} / \mathrm{cm}^{3}$ \\
\hline & $H_{1}$ & Free & $10^{-3}-10^{3} H_{g} / H_{p}$ \\
\hline & $m_{r}$ & Fixed & Ammonia ice \\
\hline & $m_{i}$ & Fixed & Ammonia ice \\
\hline & $r_{g}$ & Free & $0.05-0.5 \mu m$ \\
\hline & $\sigma_{g}$ & Fixed & 0.1 \\
\hline \multirow{10}{*}{ Tropospheric Haze } & $P_{3}$ & Free & 10-200 mbar \\
\hline & $P_{4}$ & Fixed & 1 bar \\
\hline & $N_{2}$ & Free & $10^{-1}-10^{2}$ part $/ \mathrm{cm}^{3}$ \\
\hline & $H_{2}$ & Free & $10^{-3}-10^{3} H_{g} / H_{p}$ \\
\hline & $\varpi_{02}(\mathrm{BL} 1)$ & Free & $0.5-1.0$ \\
\hline & $\varpi_{02}(\mathrm{IR})$ & Fixed & 0.995 \\
\hline & $\sigma_{2}$ & Fixed & $\pi \times 10^{-8} \mathrm{~cm}^{2}$ \\
\hline & $f$ & Free & $0.5-0.99$ \\
\hline & $g_{1}$ & Free & $0.5-0.99$ \\
\hline & $g_{2}$ & Free & $-0.05--0.5$ \\
\hline \multirow{5}{*}{ Bottom Cloud } & $P_{5}$ & Fixed & $1.0 \mathrm{bar}$ \\
\hline & $P_{6}$ & Fixed & $1.4 \mathrm{bar}$ \\
\hline & $N_{3}$ & Free & $10^{0}-10^{3}$ part $/ \mathrm{cm}^{3}$ \\
\hline & $\sigma_{3}$ & Fixed & $\pi \times 10^{-6} \mathrm{~cm}^{2}$ \\
\hline & $\varpi_{03}$ & Fixed & 0.995 \\
\hline
\end{tabular}


Table 3: Comparison of 2HG parameters with Tomasko and Doose (1984)

\begin{tabular}{ccccc}
\hline Latitude & $\mathrm{f}$ & $\mathrm{g}_{1}$ & $\mathrm{~g}_{2}$ & Source \\
\hline $0-15 \mathrm{~N}$ & $0.79 \pm 0.04$ & $0.75 \pm 0.09$ & $-0.33 \pm 0.05$ & All wavelengths - This work \\
$40-70 \mathrm{~N}$ & $0.88 \pm 0.04$ & $0.83 \pm 0.04$ & $-0.31 \pm 0.07$ & All wavelengths - This work \\
$0-70 \mathrm{~N}$ & $0.85 \pm 0.06$ & $0.81 \pm 0.07$ & $-0.31 \pm 0.07$ & All wavelengths - This work \\
\hline $7-11 \mathrm{~S}$ & 0.763 & 0.620 & -0.294 & $\mathrm{R}-\mathrm{T} \& \mathrm{D}(1984)$ \\
$15-17 \mathrm{~S}$ & 0.768 & 0.603 & -0.302 & $\mathrm{R}-\mathrm{T} \& \mathrm{D}(1984)$ \\
\hline $7-11 \mathrm{~S}$ & 0.920 & 0.710 & -0.317 & $\mathrm{~B}-\mathrm{T} \& \mathrm{D}(1984)$ \\
$15-17 \mathrm{~S}$ & 0.764 & 0.870 & -0.116 & $\mathrm{~B}-\mathrm{T} \& \mathrm{D}(1984)$ \\
\hline
\end{tabular}



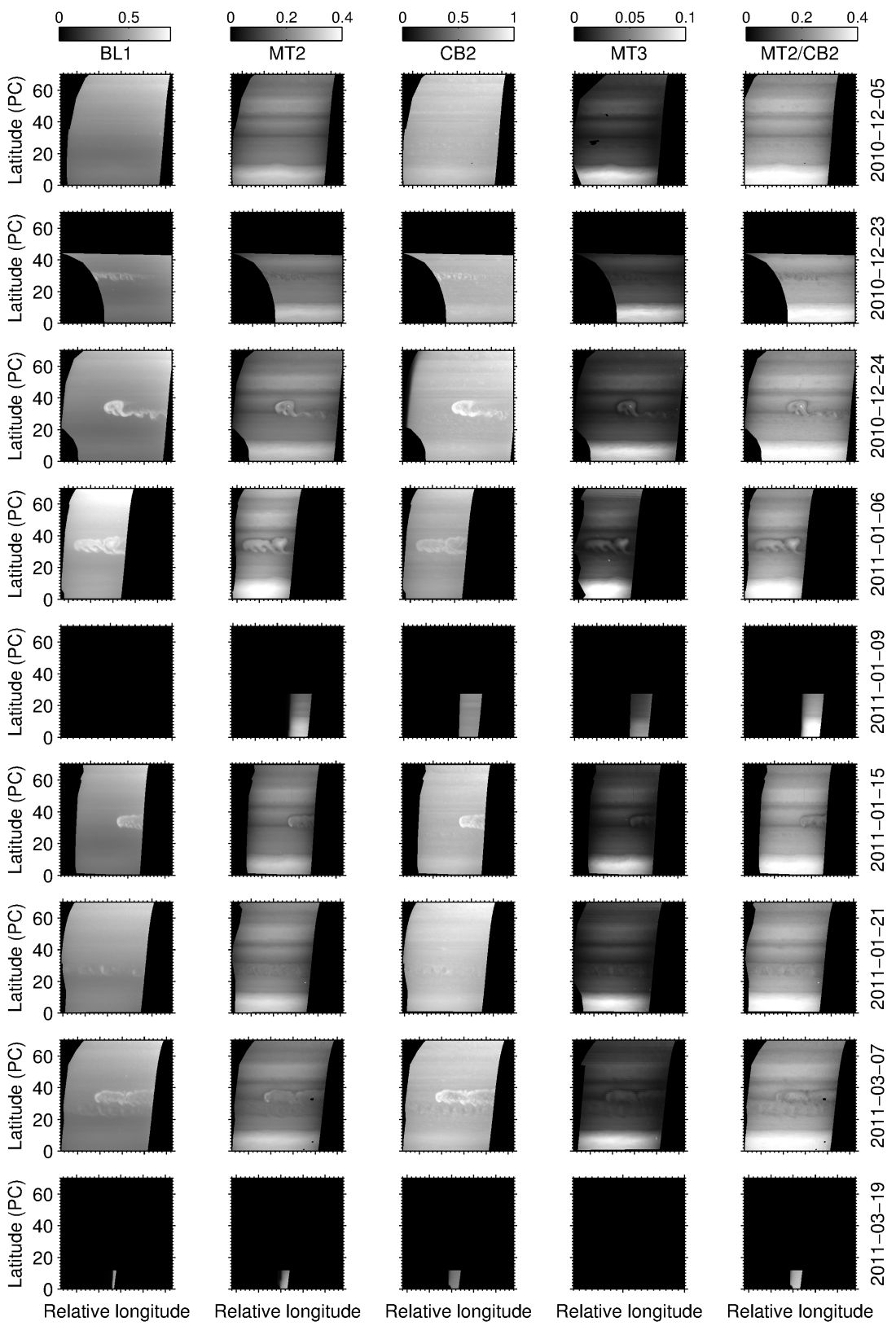

Figure 1: Cylindrically projected maps for the three selected filters and MT2/CB2 ratio dates before March, 2011. All maps are oversampled to a resolution of $0.1^{\circ}$ per pixel. Each map covers $100^{\circ}$ in longitude and spans from $0^{\circ}$ to $70^{\circ}$ North. All maps are calibrated in absolute reflectivity and Lambert corrected for limb-darkening. The reflectivity has been scaled in the cases with most extreme phase angles (January 9 and March 19) to fit the same grayscale levels. 



Figure 2: Same as figure 1 for the rest of dates. 

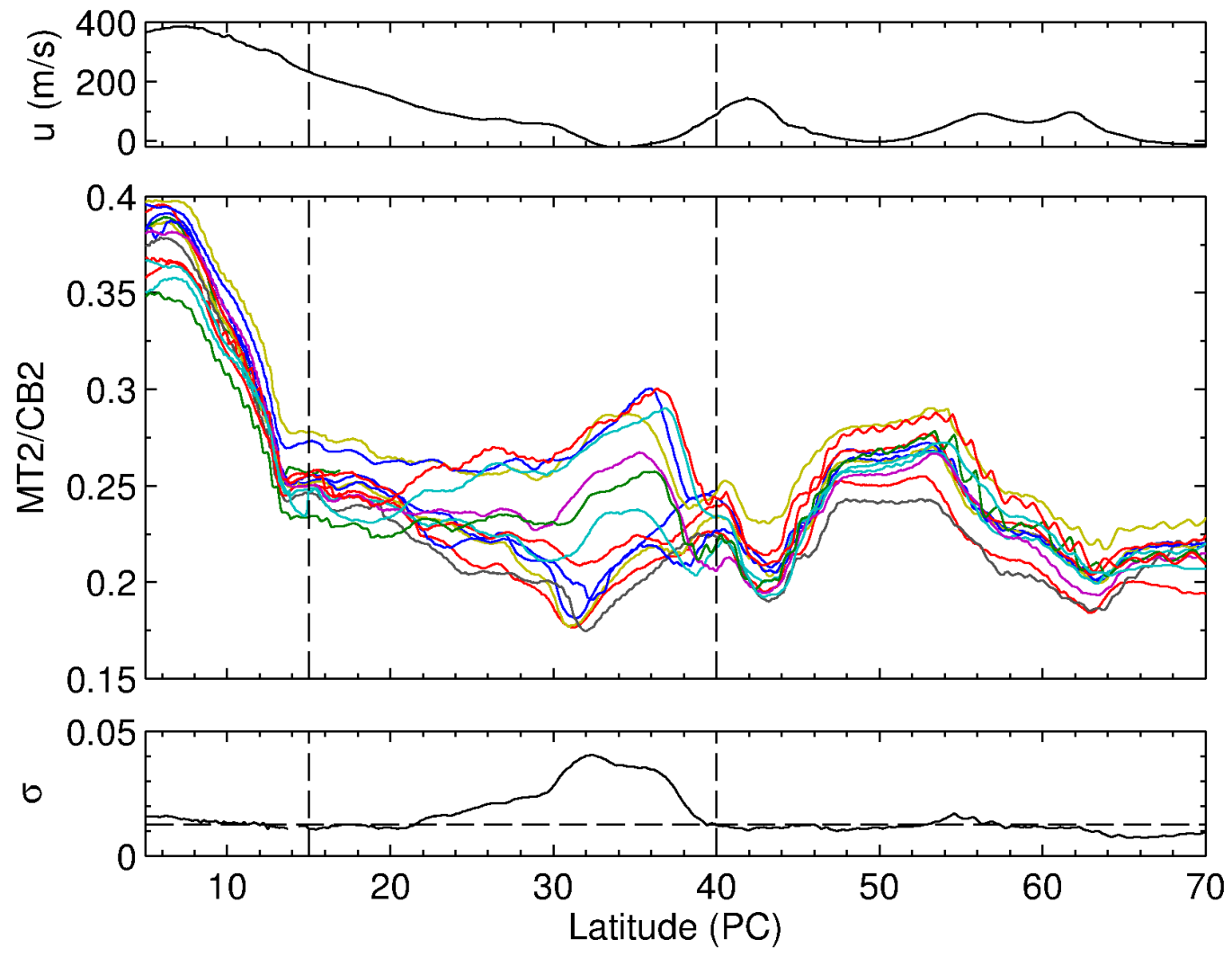

Figure 3: (up) Zonal wind speeds $u$ as a function of latitude from García-Melendo et al. (2011). (middle) Zonal means of the MT2/CB2 ratio for all dates with phase angles from $20^{\circ}$ to $100^{\circ}$. (bottom) Standard deviation $\sigma$ of all scans as a function of latitude. The horizontal dashed line shows the average of $\sigma$ for the region analyzed in this work. The area between the vertical dashed lines indicates the excluded region. 


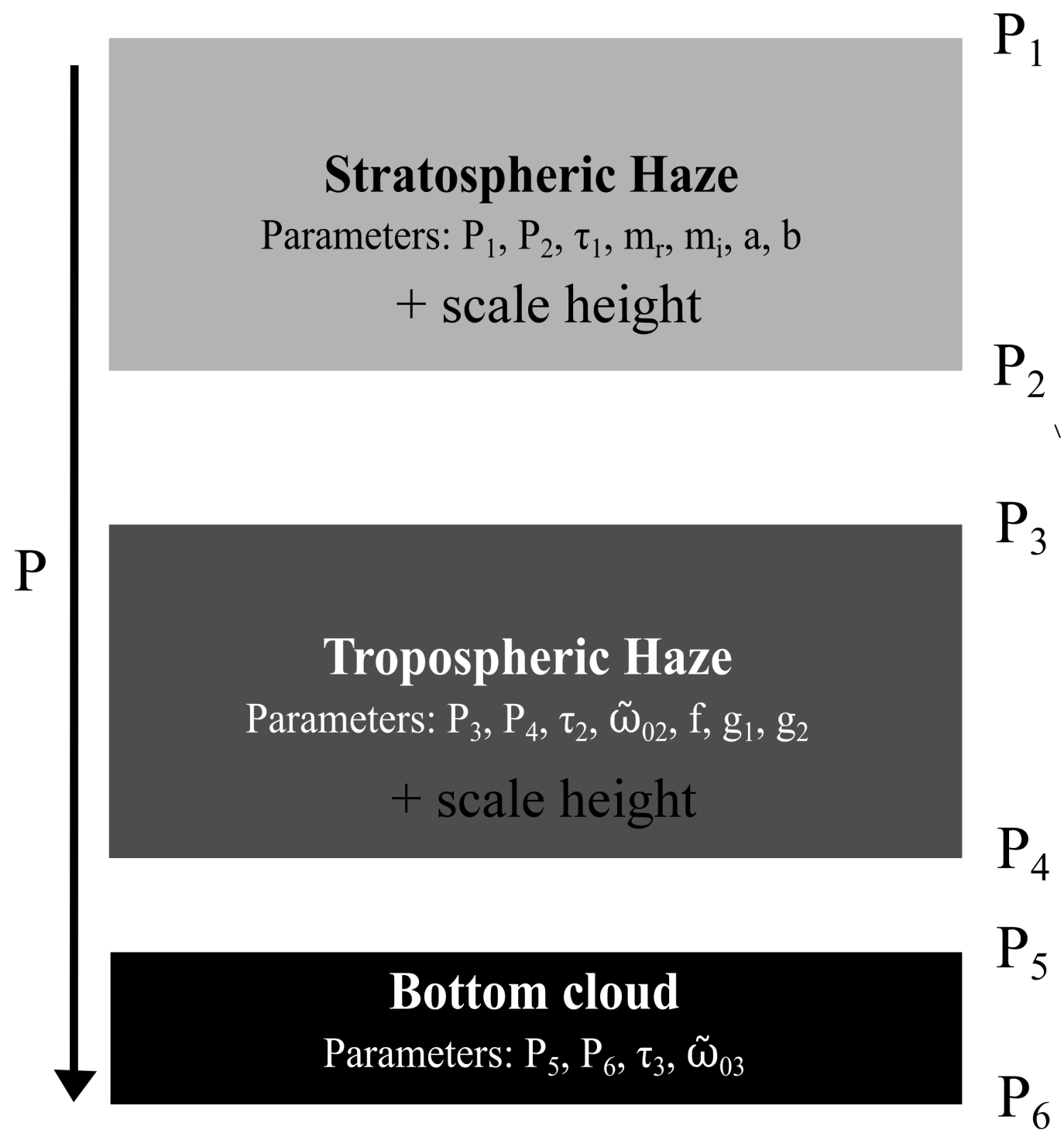

Figure 4: Scheme of the vertical cloud structure model used in this work and the parameters required to define it. All haze and cloud layers are assumed to have constant particle number density between boundary pressure levels. The particle number densities of the hazes decay exponentially above the top level with the corresponding scale height. 

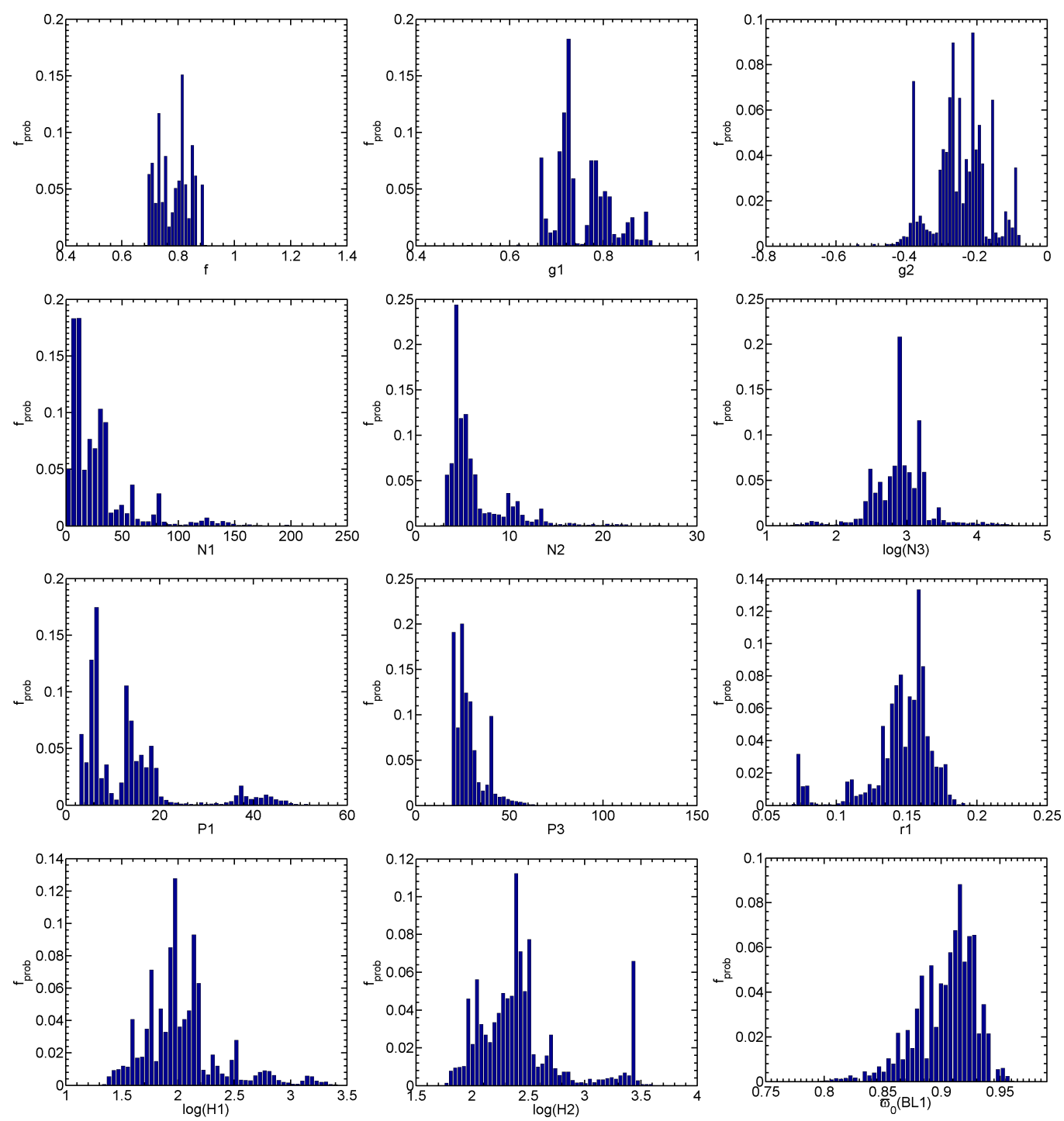

Figure 5: Histograms showing the relative frequency of each model parameter weighted by the probability of the model of being exact as explained in the text. Higher values of relative frequency implies a greater probability of this value to be correct. 

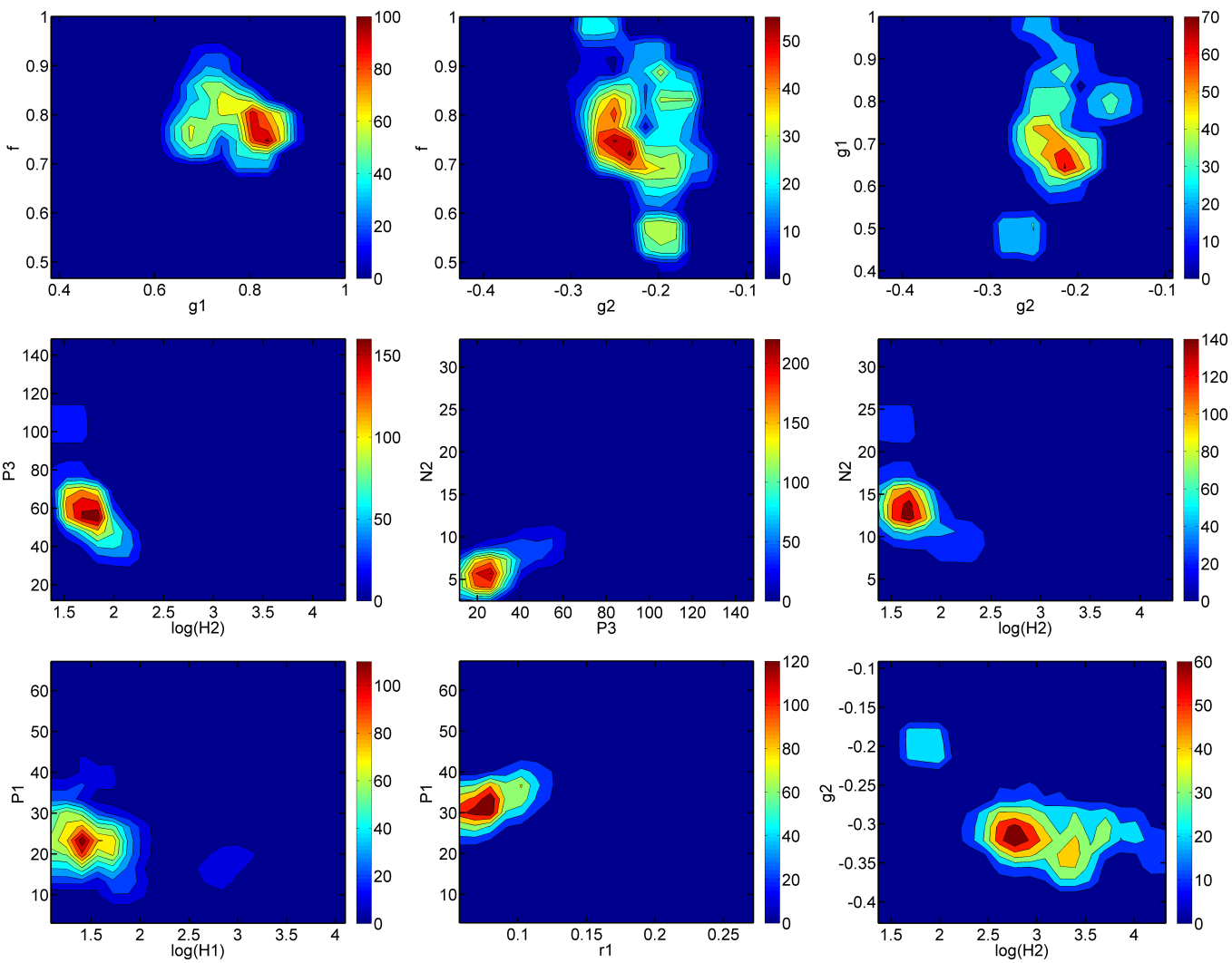

Figure 6: Density of models (weighted by the probability) for some combinations of the free parameters. Higher values in a given region of the free parameter space imply a bigger probability of the solution to be there. 


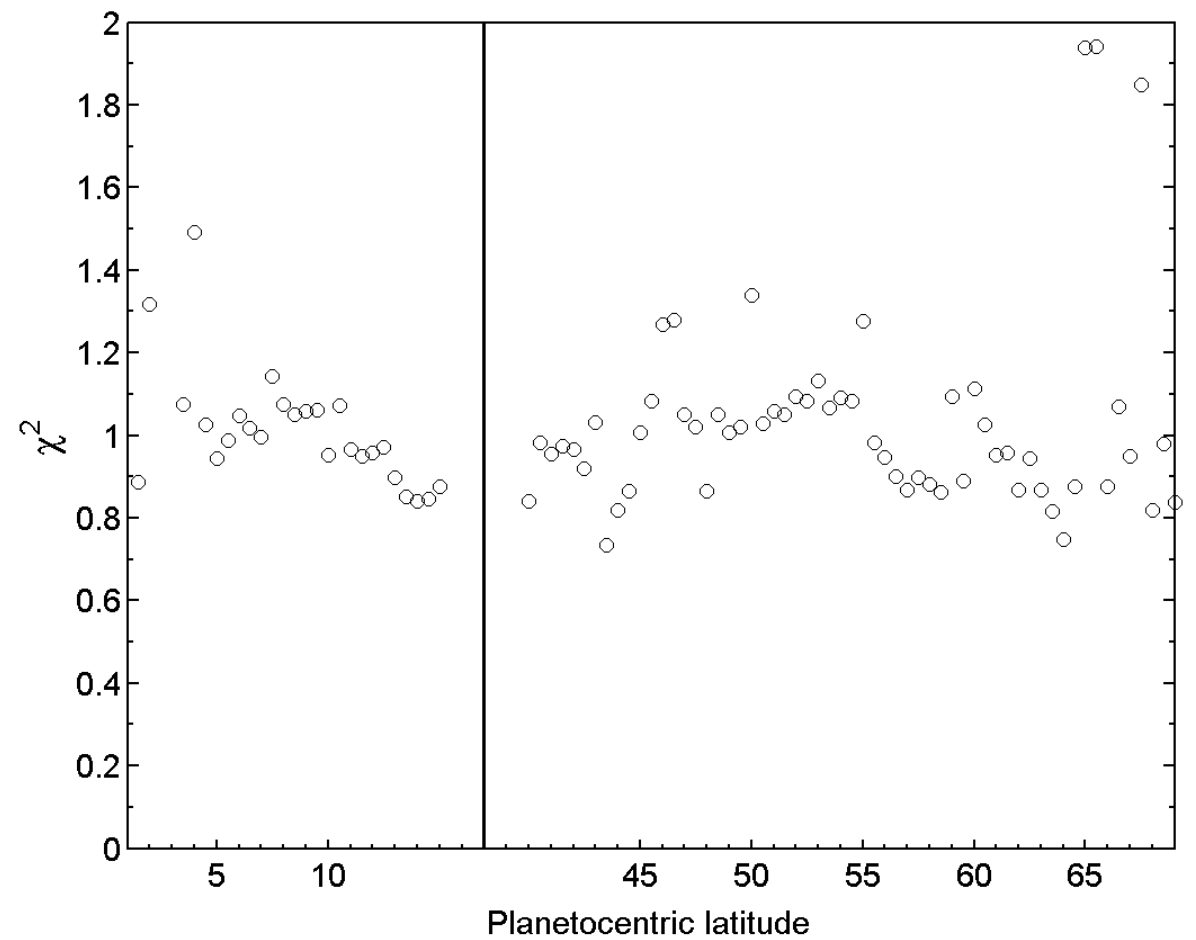

Figure 7: Reduced $\chi^{2}$ (mean for every point and wavelength) for all latitudes. 

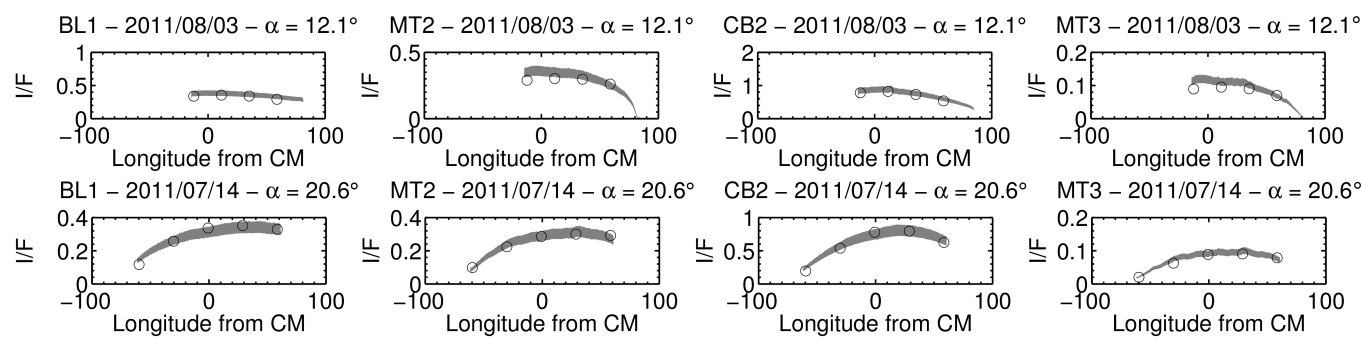

$-100 \quad 000$
Longitude from $\mathrm{CM}^{100}$
$\mathrm{BL} 1-2011 / 08 / 06-\alpha=28.2$

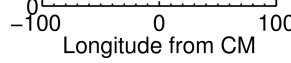

Longitude from $\mathrm{CM}^{100}$
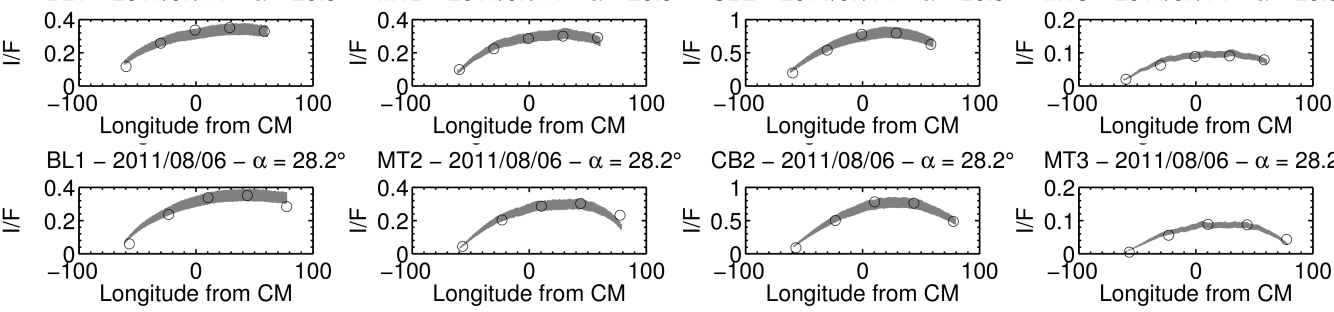

MT3 $-2011 / 08 / 06-\alpha=28.2^{\circ}$

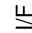

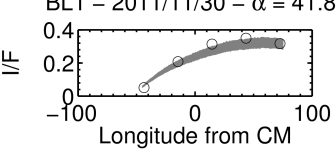

MT2 $-2011 / 11 / 30-\alpha=41.8^{\circ}$
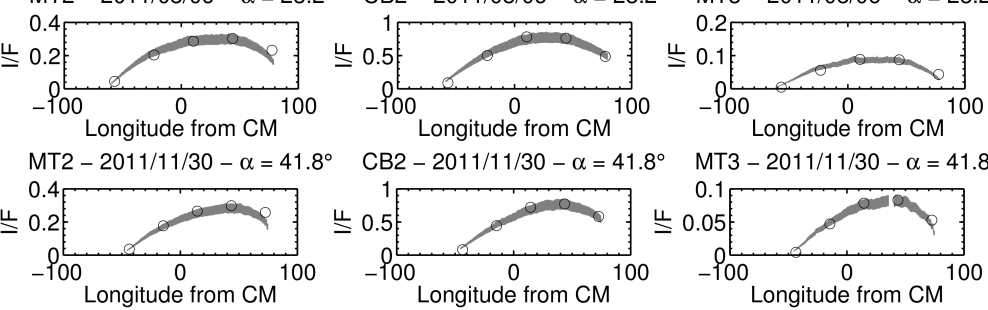

MT3 $-2011 / 11 / 30-\alpha=41.8^{\circ}$

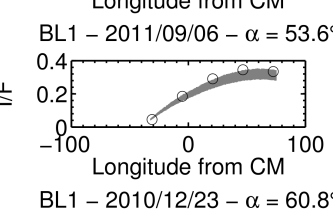

-100 Longitude from $\mathrm{CM}^{100}$
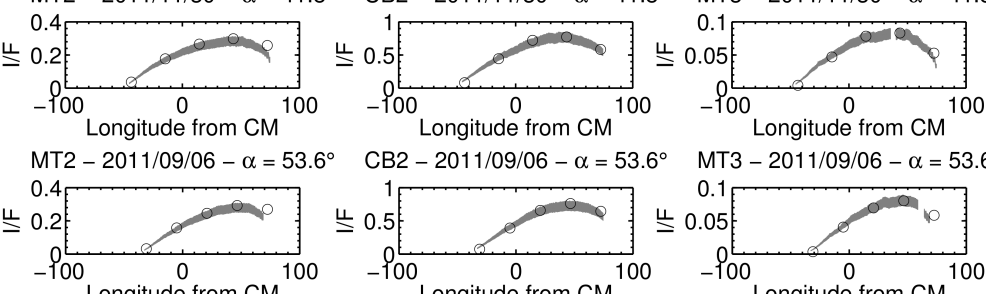

Longitude from $\mathrm{CM}$
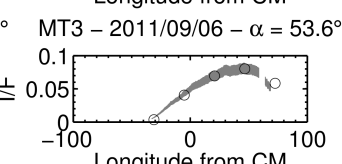

Longitude from CM
MT2 $-2010 / 12 / 23-\alpha=60.8^{\circ}$

Longitude from $\mathrm{CM}^{100}$
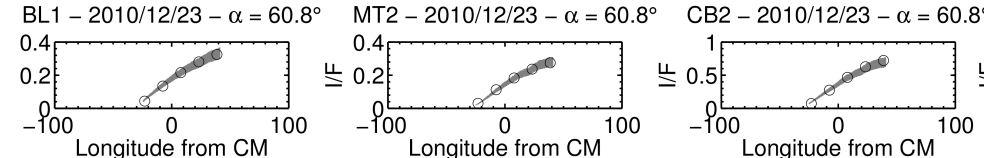

Longitude from $\mathrm{CM}$

$\stackrel{4}{=}$

Longitude from $\mathrm{CM}$

BL1 - 2010/12/24 - $\alpha=71.2^{\circ}$

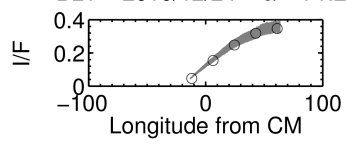

MT2 - 2010/12/24 - $\alpha=71.2$

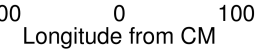

ㄱT3 $-2010 / 12 / 23-\alpha=60.8^{\circ}$

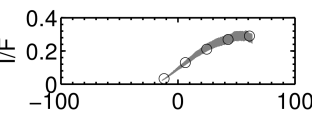

CB2 $-2010 / 12 / 24-\alpha=71$
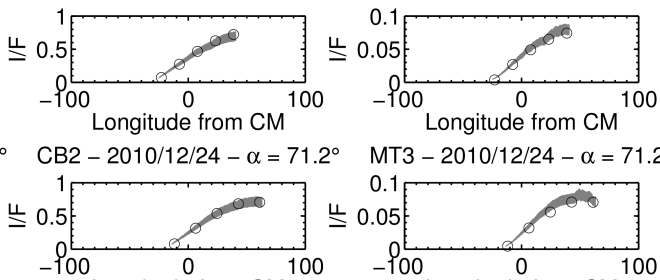

MT3 - 2010/12/24 -
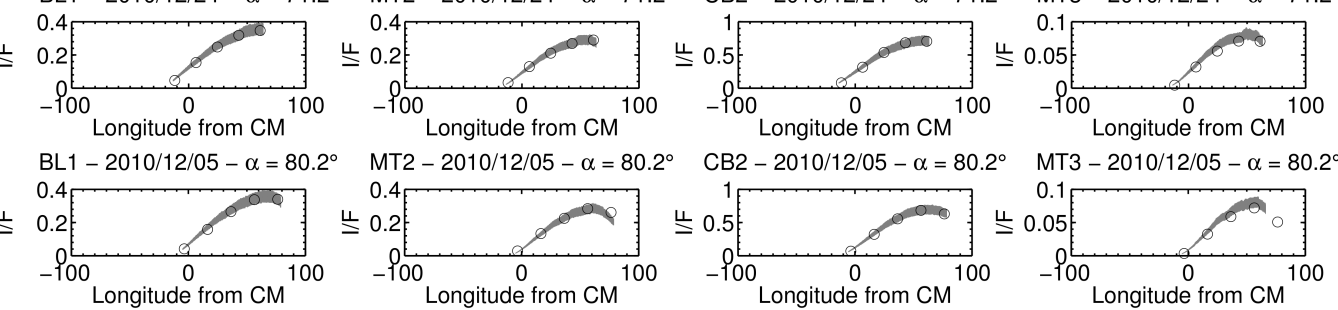

Longitude from $\mathrm{CM}{ }^{100}$
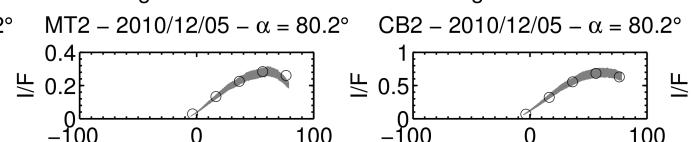

MT3 - 2010/12/05 $-\alpha=80.2^{\circ}$
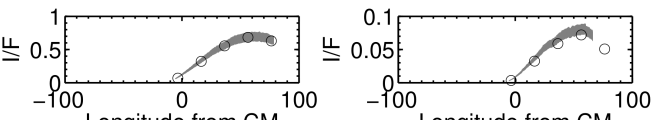

BL1 $-2011 / 01 / 21-\alpha=95.4^{\circ}$

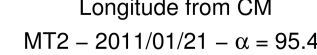

Longitude from $\mathrm{CM}^{100}$

Longitude from $\mathrm{CM}^{100}$
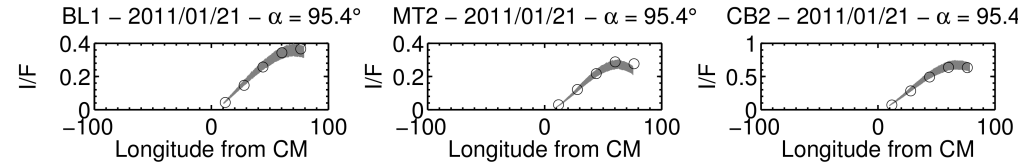

․ $\mathrm{MT3}-2011 / 01 / 21-\alpha=95.4^{\circ}$
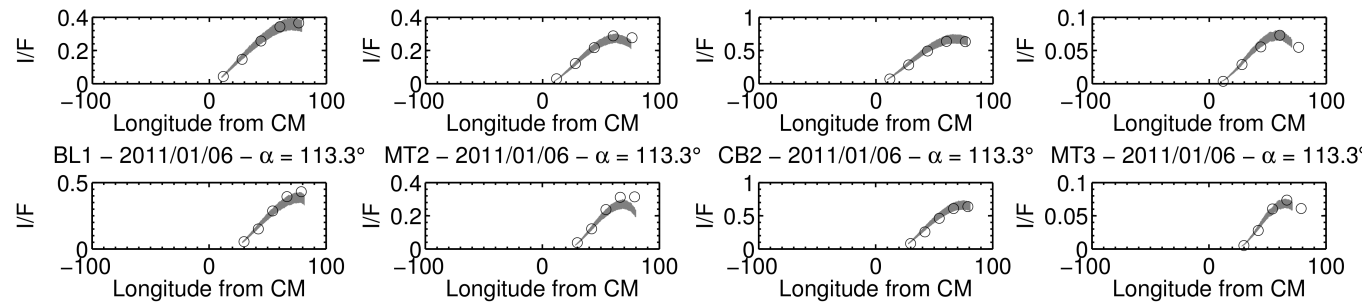

BL1 $-2011 / 01 / 09-\alpha=147.0^{\circ}$
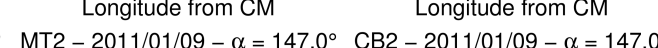

-100 Longitude from $\mathrm{CM}^{100}$
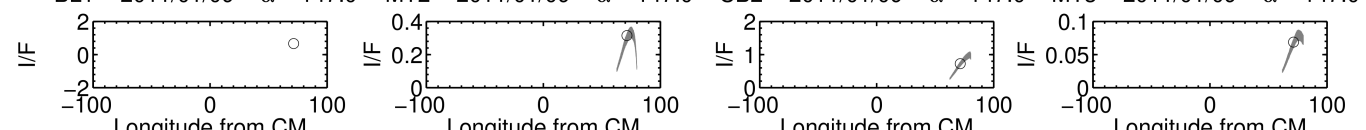

BL1 $-2011 / 03 / 19-\alpha=158.5^{\circ} \quad$ MT2 $-2011 / 03 / 19-\alpha=158.5^{\circ}$ CB2 $-2011 / 03 / 19-\alpha=158.5^{\circ}$ MT3 $-2011 / 03 / 19-\alpha=158.5^{\circ}$
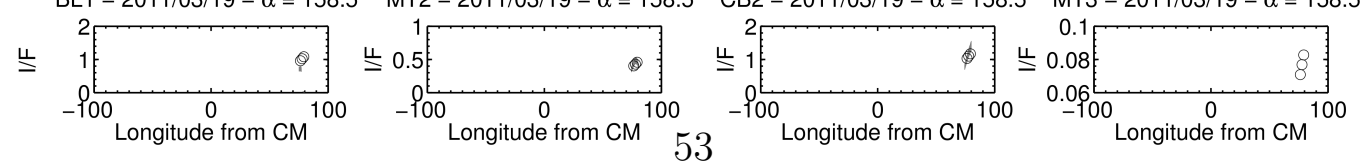

Figure 8: Comparison between observed reflectivity (grey) and best-fitting models (black circles) for some selected dates at $5^{\circ} \mathrm{N}$. Dates have been chosen to cover the whole phase angle range at similar intervals and they are shown in increasing values of $\alpha$ from top to bottom. 

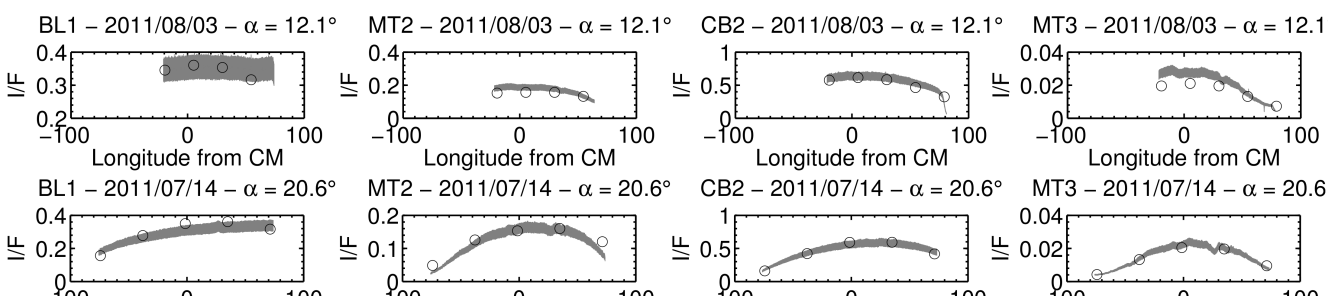

MT2 - 2011/07/14 - $\alpha=20.6^{\circ}$

CB2 $-2011 / 07 / 14-\alpha=20.6^{\circ} \quad$ MT3 $-2011 / 07 / 14-\alpha=20.6^{\circ}$
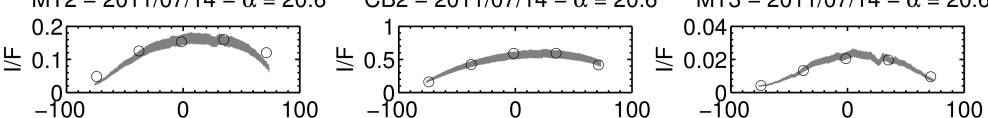

Longitude from CM
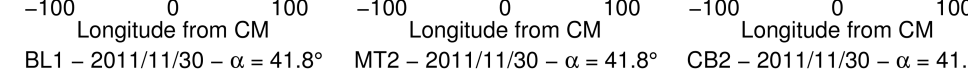

Longitude from $\mathrm{CM}^{100}$
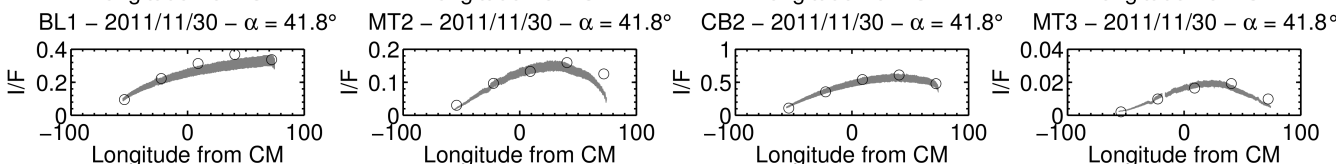

$\mathrm{BL} 1-2011 / 09 / 06-\alpha=53.6^{\circ}$

MT2 $-2011 / 09 / 06-\alpha=53.6^{\circ}$

CB2 $-2011 / 09 / 06-\alpha=53.6^{\circ} \quad$ MT3 $-2011 / 09 / 06-\alpha=53.6^{\circ}$

0.4
$\Perp$

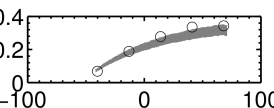

$100{ }^{0}$ Longitude from $\mathrm{CM}^{100}$
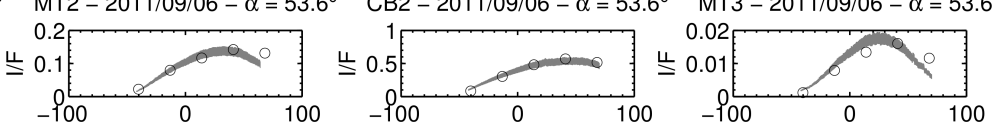

$\mathrm{BL} 1-2010 / 12 / 24-\alpha=71.2^{\circ} \quad \mathrm{MT} 2-2010 / 12 / 24-\alpha=71.2^{\circ}$
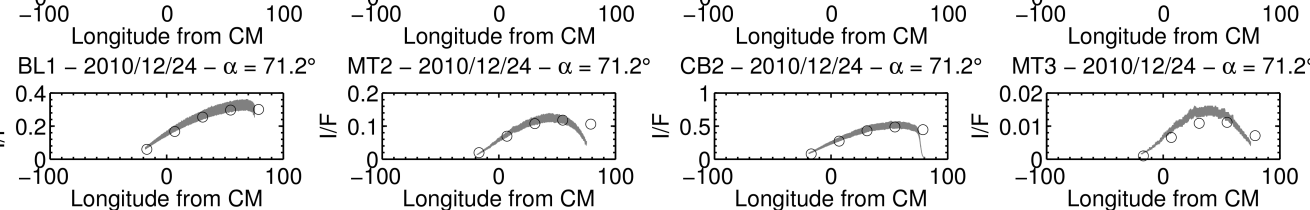

Longitude from $\mathrm{CM}$

BL1 $-2010 / 12 / 05-\alpha=80.2^{\circ}$

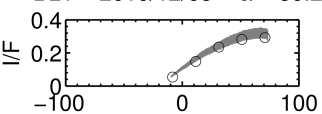

-100 Longitude from $\mathrm{CM}^{100}$

BL1 $-2011 / 01 / 21-\alpha=95.4^{\circ}$

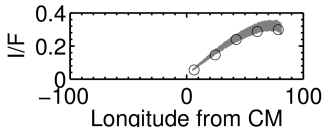

MT2 $-2010 / 12 / 05-\alpha=80$.

CB2 $-2010 / 12 / 05-\alpha=80.2^{\circ} \quad$ MT3 $-2010 / 12 / 05-\alpha=80.2^{\circ}$

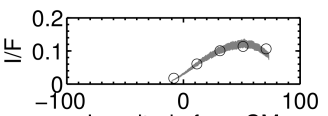

Longitude from $\mathrm{CM}$

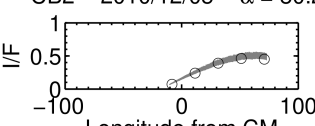

MT3 $-2010 / 12 / 24-\alpha=71.2$
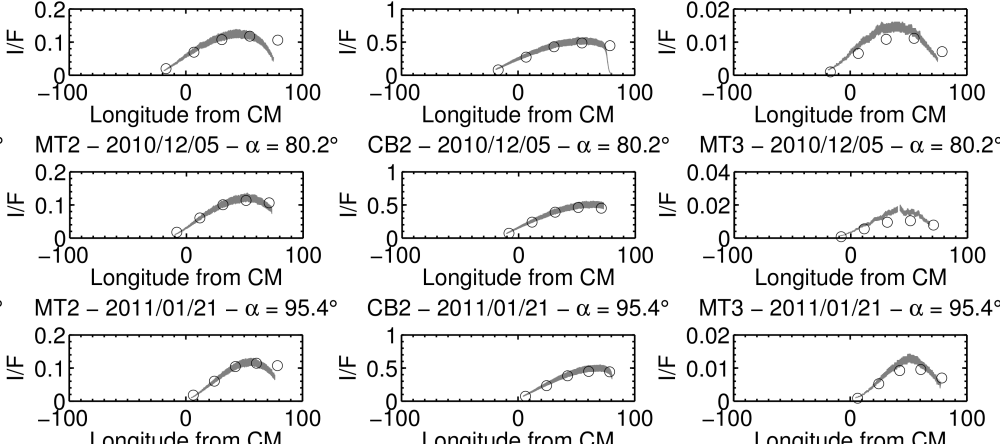

Longitude from $\mathrm{CM}$

BL1 $-2011 / 01 / 06-\alpha=113.3^{\circ}$ MT2 $-2011 / 01 / 06-\alpha=113.3^{\circ}$
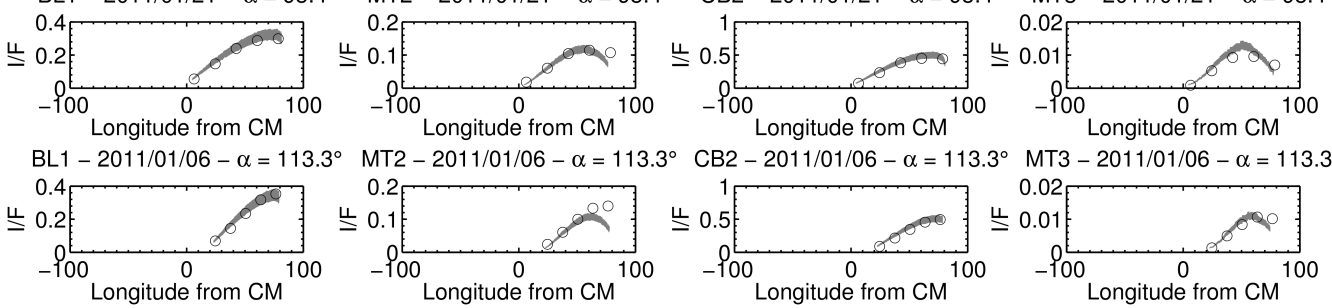

$3^{\circ}$ MT3 $-2011 / 01 / 06-\alpha=113.3^{\circ}$

Figure 9: Same as figure 8 but for $45^{\circ} \mathrm{N}$. Note the more limited phase angle coverage for this case. 

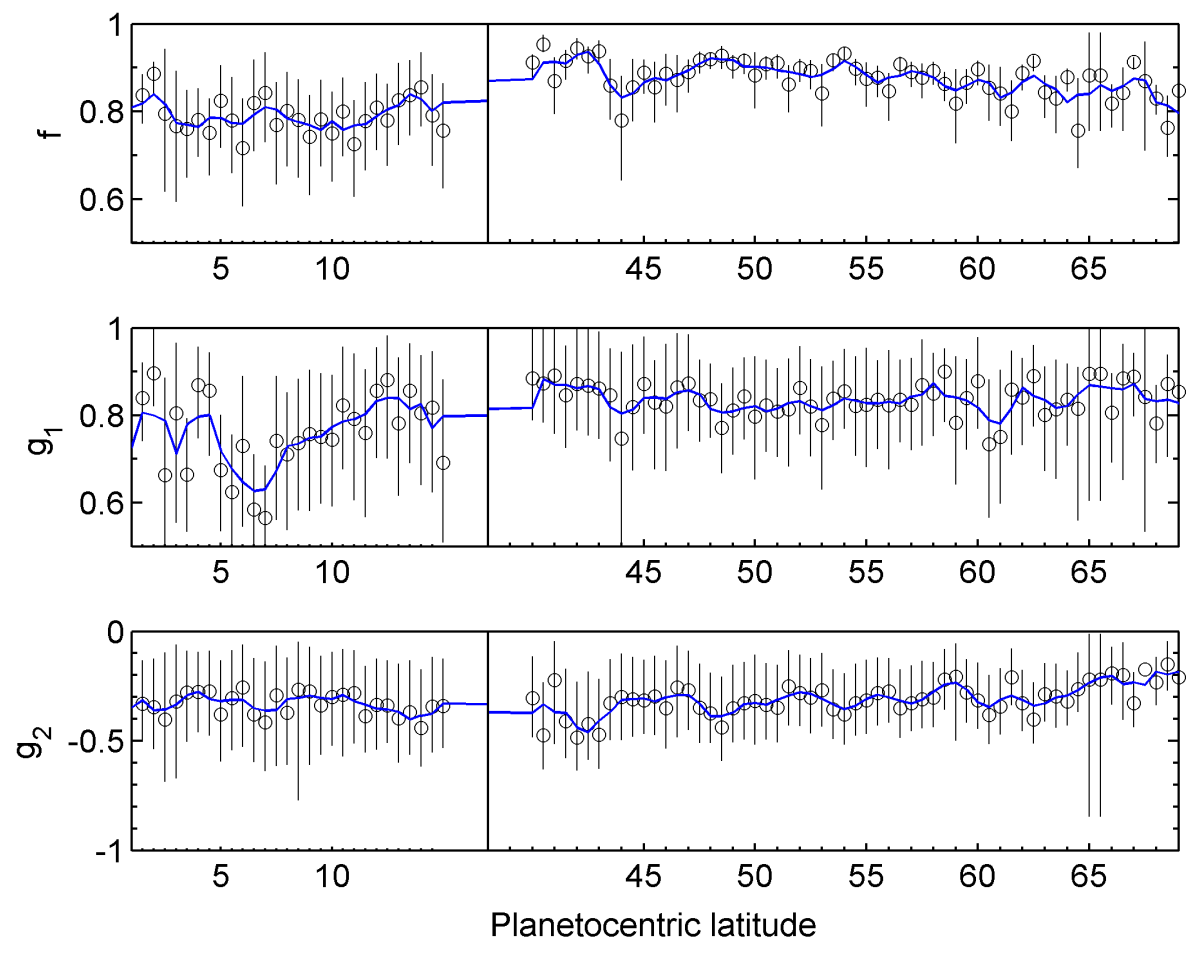

Figure 10: Optimal values of $2 \mathrm{HG}$ parameters as a function of latitude (circles) and estimated error bars. The solid line is a running average smooth in $2^{\circ}$ latitude bins. These values are valid for all the wavelengths covered in this work, as explained in the text. 


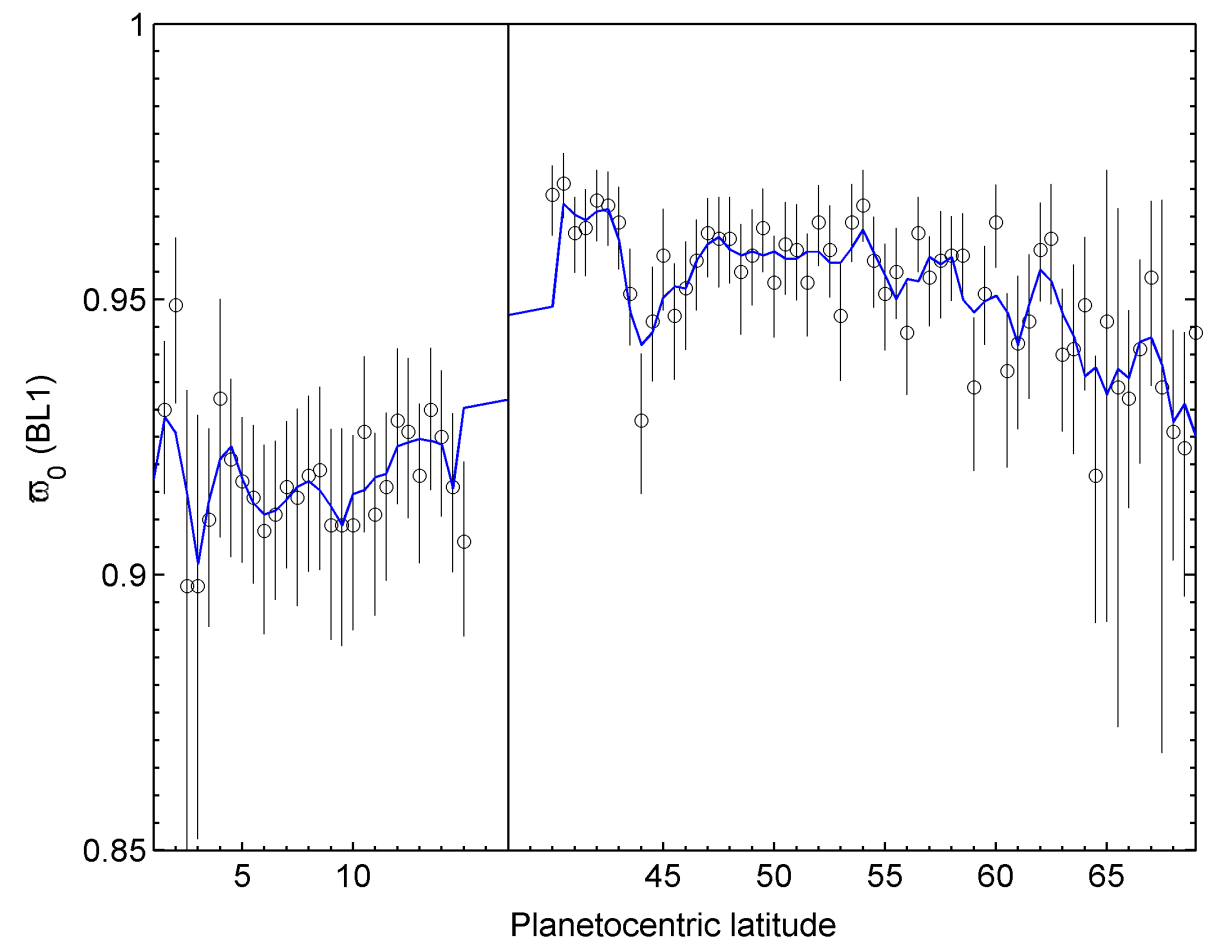

Figure 11: Single scattering albedo $\varpi_{0}$ at blue wavelengths (circles) as a function of latitude. The solid line is a running average smooth in $2^{\circ}$ latitude bins. 

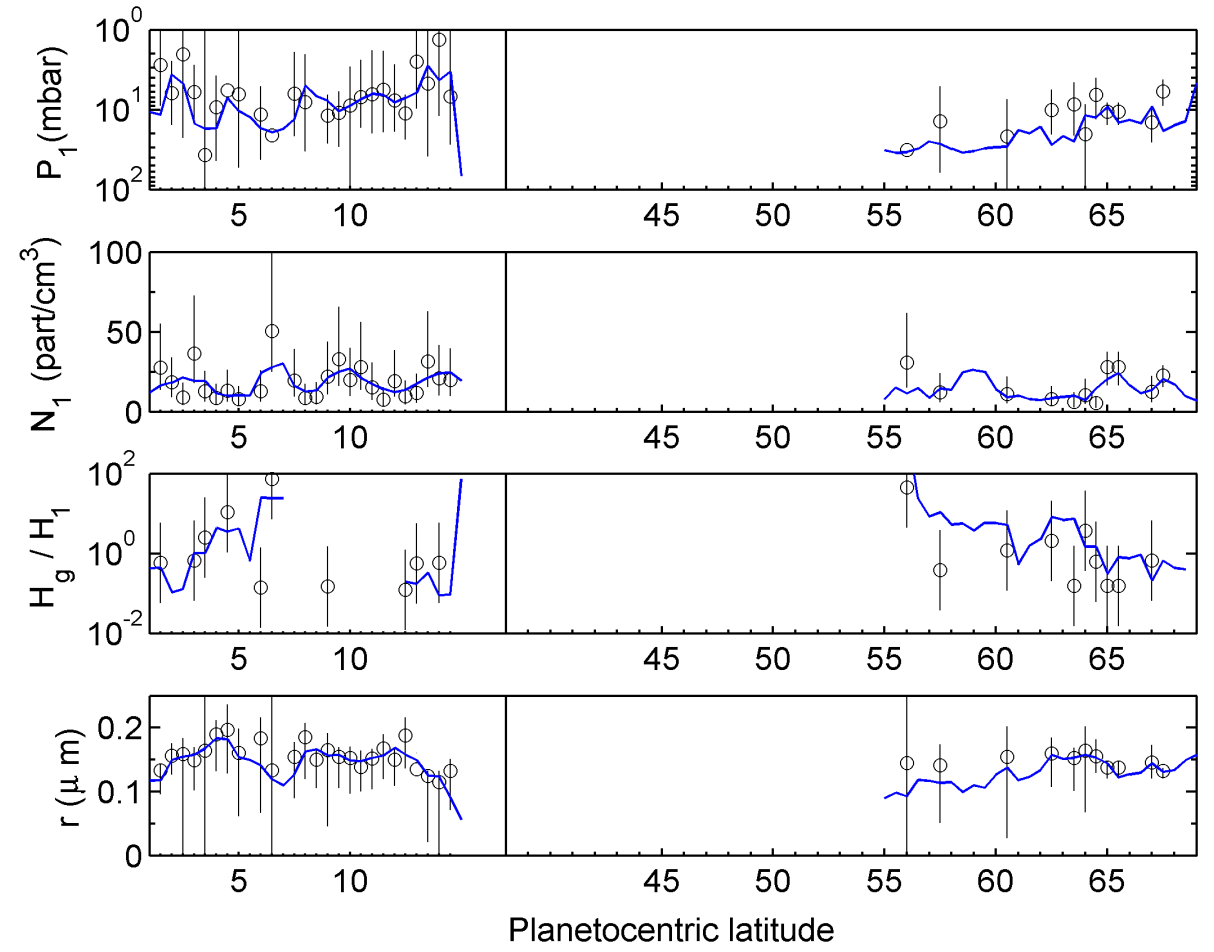

Figure 12: Best-fitting parameters for the stratospheric haze (circles). Results are shown only for the latitudes with integrated optical thickness $\tau_{s t r}>0.01$. The solid line is a running average smooth in $2^{\circ}$ latitude bins. Scale height is given in terms of the gas scale height $H_{g}$. 

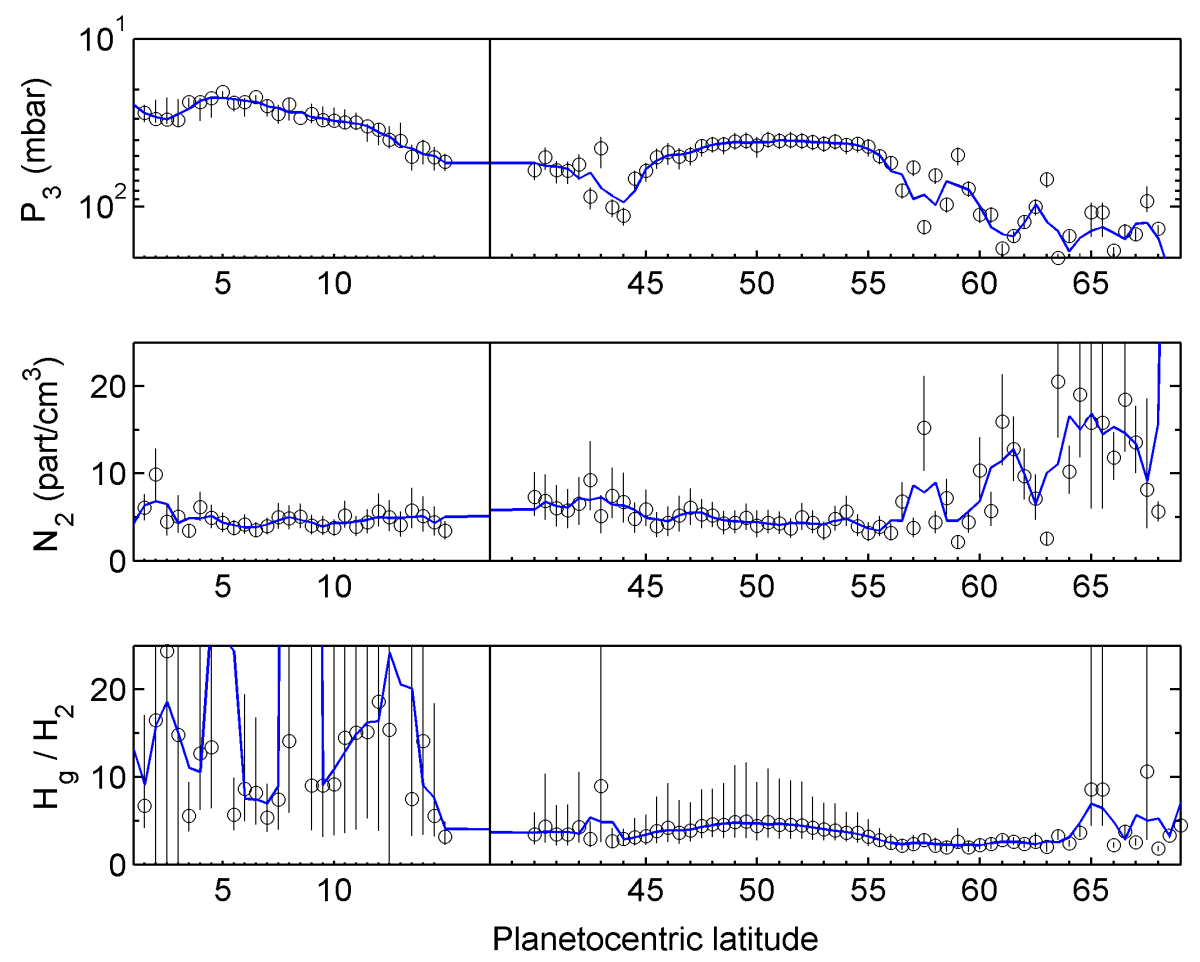

Figure 13: Best-fitting parameters for the tropospheric haze (circles). The solid line is a running average smooth in $2^{\circ}$ latitude bins. Scale height is given in terms of the gas scale height $H_{g}$. 

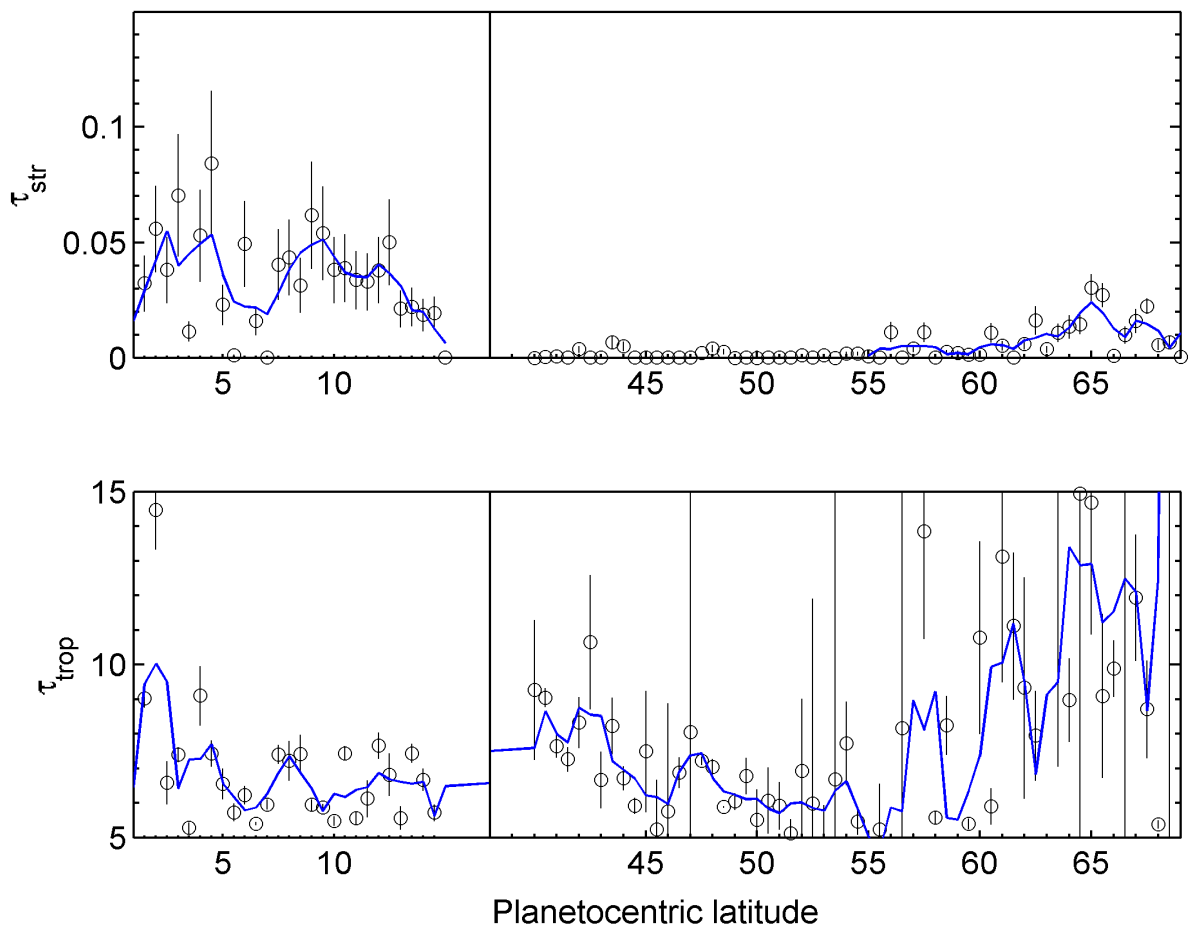

Figure 14: Total optical thickness of the stratospheric (top) and tropospheric (bottom) hazes. As defined in the text, the first provides the total optical thickness above $\mathrm{P}_{2}=100$ mbar caused by stratospheric particles and the second the total optical thickness above $\mathrm{P}_{4}=1$ bar caused by the tropospheric particles. 


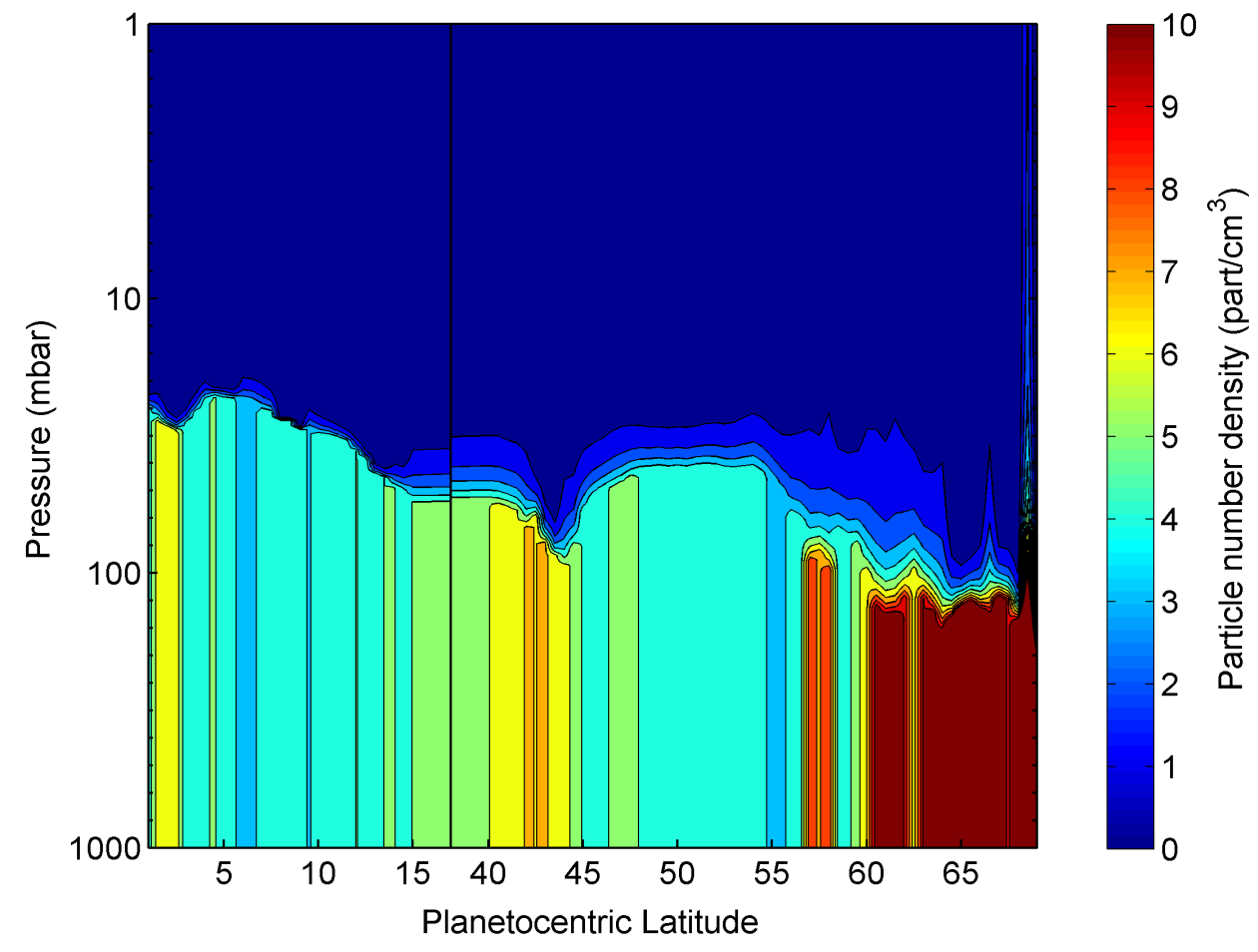

Figure 15: Tropospheric particle number density as a function of latitude and pressure. This plot has been constructed using the smoothed version of the retrievals shown in the previous figures, using $2^{\circ}$ latitude bins. Stratospheric aerosols are not shown in this figure. Note that number density was forced to be constant between limiting pressures $\mathrm{P}_{3}$ and $\mathrm{P}_{4}$ $=1$ bar by model construction. 

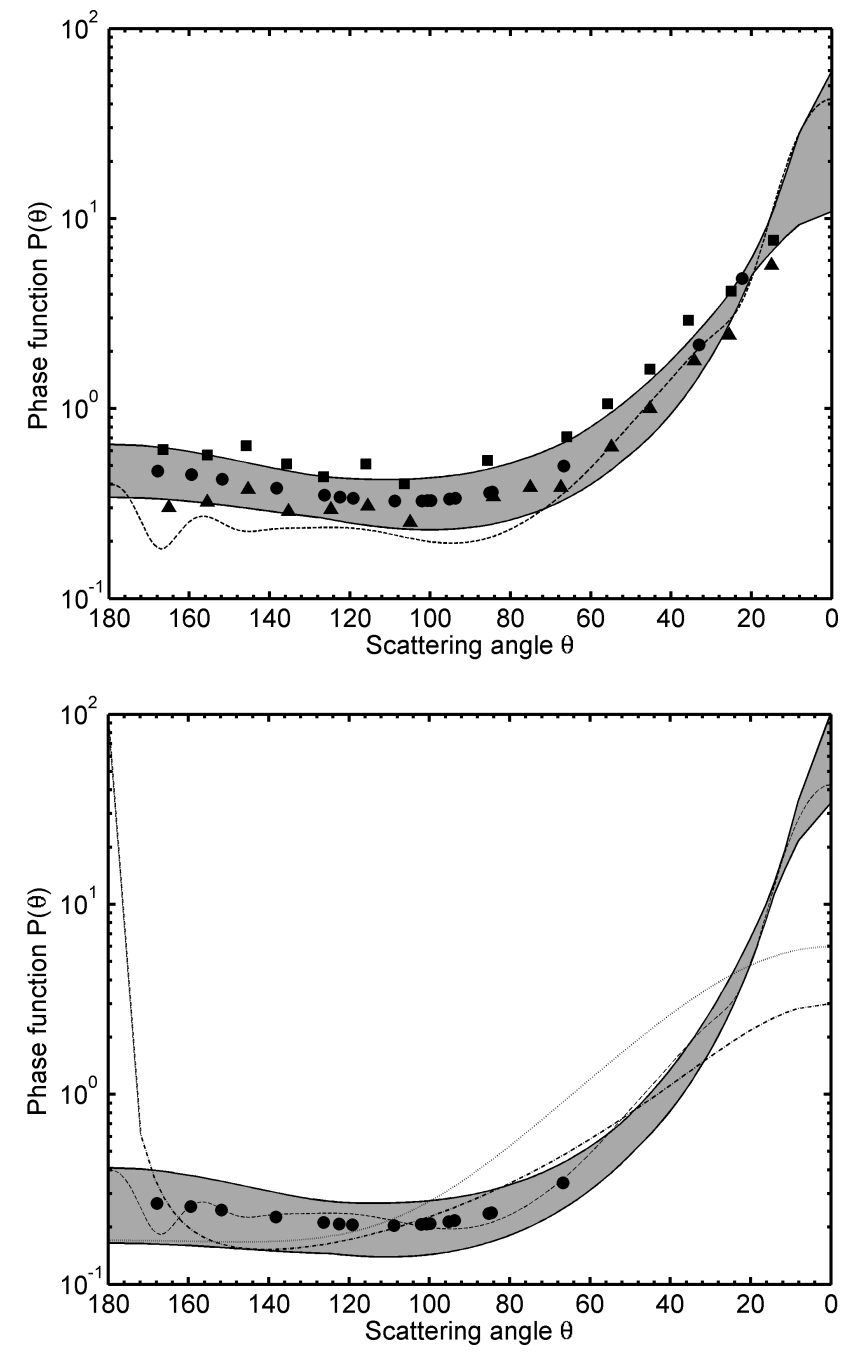

Figure 16: (top) Phase function of the EZ particles at the actual phase angles covered in this work with the nominal values of table 3 (circles) and extrapolated to all possible scattering angles with the retrieved error bars (grey area). This is compared with the phase function of $10 \mu \mathrm{m}$ ice crystals grown in laboratory (Pope et al., 1992) at blue (triangles) and red (squares) wavelengths. Dashed line is used for spheroids of $1 \mu \mathrm{m}$ mean radius and aspect ratio of 2/3. (bottom) Same for the non equatorial particles. Dotted line is used for particles $0.25 \mu \mathrm{m}$ mean radius and aspect ratio of $1 / 3$, marginally compatible at intermediate scattering angles. The dashed-dotted line is used for an alternative double Henyey-Greenstein phase function $\left(f=0.5, g_{1}=0.5, g_{2}=-0.95\right)$ compatible with phase function values at the observed scattering ongles. Note that this phase function implies a heavy opposition surge. A scattering angle of $0^{\circ}$ means forward scattering, while $180^{\circ}$ means backward scattering. 

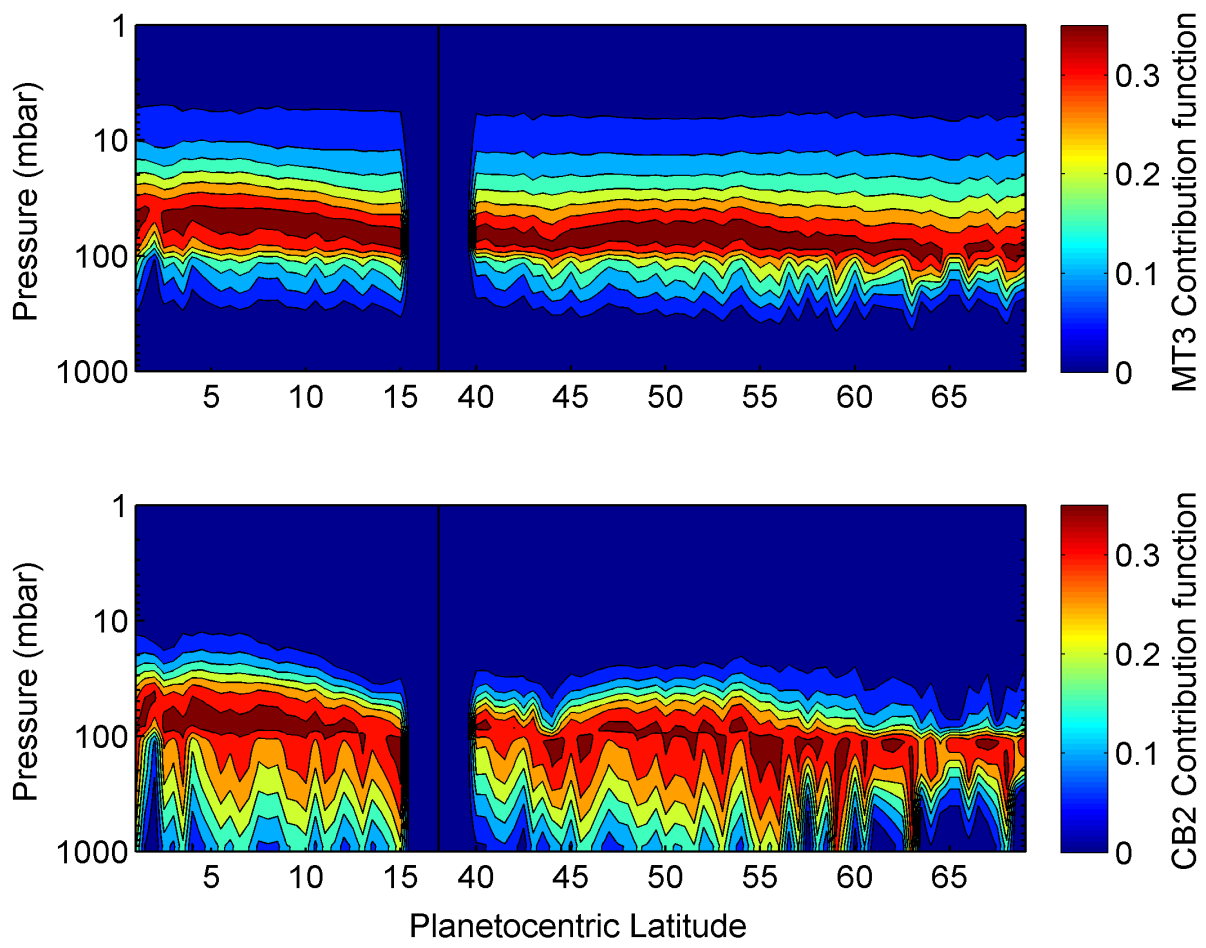

Figure 17: Contribution function for MT3 (top) and CB2 (bottom). These include gas absorption by $\mathrm{CH}_{4}$ and particle scattering using the values retrieved in the previous sections. Please note that discontinuities are produced by the particle distribution in the atmospheric model. 